\title{
Constrained Subpixel Target Detection for Remotely Sensed Imagery
}

\author{
Chein-I Chang, Senior Member, IEEE, and Daniel C. Heinz, Student Member, IEEE
}

\begin{abstract}
Target detection in remotely sensed images can be conducted spatially, spectrally or both. The difficulty of detecting targets in remotely sensed images with spatial image analysis arises from the fact that the ground sampling distance is generally larger than the size of targets of interest in which case targets are embedded in a single pixel and cannot be detected spatially. Under this circumstance target detection must be carried out at subpixel level and spectral analysis offers a valuable alternative. In this paper, the problem of subpixel spectral detection of targets in remote sensing images is considered, where two constrained target detection approaches are studied and compared. One is a target abundance-constrained approach, referred to as nonnegatively constrained least squares (NCLS) method. It is a constrained least squares spectral mixture analysis method which implements a nonnegativity constraint on the abundance fractions of targets of interest. Another is a target signature-constrained approach, called constrained energy minimization (CEM) method. It constrains the desired target signature with a specific gain while minimizing effects caused by other unknown signatures. A quantitative study is conducted to analyze the advantages and disadvantages of both methods. Some suggestions are further proposed to mitigate their disadvantages.
\end{abstract}

Index Terms-Constrained energy minimization (CEM), nonnegatively constrained least squares (NCLS), orthogonal subspace projection (OSP).

\section{ACRONYMS}

ANC Abundance nonnegativity constraint.

ASC Abundance sum-to-one constraint.

AVIRIS Airborne visible/infrared imaging spectrometer.

CEM Constrained energy minimization.

FCLS Fully constrained least squares.

FIR Finite impulse response.

FNNLS Fast NNLS.

FNNLSb Second version of FNNLS.

HYDICE Hyperspectral digital imagery collection experiment.

LSE Least equares error.

LSMA Linear spectral mixture analysis.

MVDR Minimum variance distortionless response.

NCLS Nonnegatively constrained least squares.

NNLS Nonnegative least squares.

OSP Orthogonal subspace projection.

SCLS Sum-to-one constrained least squares.

UCEM Unsupervised constrained energy minimization.

Manuscript received June 29, 1999; revised October 18, 1999.

The authors are with the Remote Sensing Signal and Image Processing Laboratory, Department of Computer Science and Electrical Engineering, University of Maryland Baltimore County, Baltimore, MD 21250 USA (e-mail: cchang@umbc.edu).

Publisher Item Identifier S 0196-2892(00)03929-2.
UNCLS Unsupervised nonnegatively constrained least squares.

UOSP Unsupervised orthogonal subspace projection.

\section{INTRODUCTION}

Q UBPIXEL target detection has received considerable interest in remote sensing image processing due to the significantly improved spectral resolution by recent advances of remote sensing instruments such as the AVIRIS and HYDICE sensor. The need for subpixel spectral detection in remotely sensed imagery arises from the fact that the ground sampling distance is generally larger than the size of targets of interest. In this case targets are embedded in a single pixel and cannot be detected spatially. As a result, traditional spatial analysis-based image processing techniques are not applicable. One must rely on and take advantage of the targets' spectral properties such as spectral contrast, variability, similarity and discriminability to be able to detect targets effectively at subpixel level. One general approach studied in the past [1], [2] was based on spectral mixture analysis [3]-[11]. In this paper, this problem is investigated and two different approaches are proposed for subpixel spectral detection of targets. One is a target abundance-constrained approach, referred to as NCLS method. It is a constrained least squares spectral mixture analysis method, which implements a nonnegativity constraint on the abundance fractions of targets of interest. The second approach is different from the NCLS method and can be derived from linear adaptive beamforming in sensor array processing. It is a target signature-constrained approach, called the CEM method, which was first proposed in [12]. It constrains the desired target signature using a specific gain while minimizing effects resulting from other unknown signatures.

In LSMA, a linear mixture model is used. Suppose that $\boldsymbol{m}_{1}, \boldsymbol{m}_{2}, \ldots, \boldsymbol{m}_{p}$ are target spectral signatures resident in a multispectral/hyperspectral image pixel vector $\boldsymbol{r}$, and $\alpha_{1}, \alpha_{2}, \ldots, \alpha_{p}$ are their associated abundance fractions within $\boldsymbol{r}$. A linear mixture model of $\boldsymbol{r}$ makes use of a mixing equation to model the spectral signature of $\boldsymbol{r}$ as a linear combination of $\boldsymbol{m}_{1}, \boldsymbol{m}_{2}, \ldots, \boldsymbol{m}_{p}$ with appropriate abundance fractions specified by $\alpha_{1}, \alpha_{2}, \ldots, \alpha_{p}$. In general, two constraints must be imposed on this model to yield an optimal solution. These are the ASC, $\sum_{j=1}^{p} \alpha_{j}=1$ and the ANC, $\alpha_{j} \geq 0$ for all $1 \leq j \leq p$. An LSMA-based FCLS method was studied in [9], [13], [14], with the goal aiming at quantifying materials present in a pixel vector $\boldsymbol{r}$. In this case, the abundance fractions $\alpha_{1}, \alpha_{2}, \ldots, \alpha_{p}$ must be estimated accurately to reflect the true abundance fractions of different materials, a task that many unconstrained or partially constrained LSMA methods 
cannot accomplish. However, from a target detection's point of view, whether or not the estimated amount of the target abundance is accurate may not be essential. As long as the estimated abundance fractions of desired target pixel vectors can distinguish themselves from their surrounding pixel vectors, the targets can be detected effectively even if the abundance fractions of LSMA do not satisfy ASC or ANC. Such target detectability was demonstrated by the unconstrained OSP methods in [15], [16]. Accordingly, for target detection purposes, an LSMA-based fully constrained least squares method sometimes may not be as effective as partially constrained or unconstrained LSMA-based methods. This is because a fully constrained method requires both $\mathrm{ASC}$ and $\mathrm{ANC}$, and the corresponding abundance fraction estimates are confined to the range of $[0,1]$, which may limit its target detection capability.

Two LSMA-based partially constrained least squares methods have been considered in the past: the SCLS [8], [13] method and the NCLS method [17], [18]. The SCLS imposes the ASC, while ignoring the ANC. On the contrary, the NCLS implements the ANC on targets of interest while discarding the ASC. As a result, both methods generally do not estimate target abundance fractions accurately. Nevertheless, their estimated abundance fractions can be used for target detection purposes. Since SCLS-generated abundance fractions must be summed to one, when an image scene contains many target signatures, which is the case for hyperspectral imagery, the magnitudes of the SCLS-detected target abundance fractions will be spread out. As a result, they will be relatively small in order to satisfy to the sum-to-one constraint. Therefore, the target detectability is considerably reduced. The situation become even worse if the spectra of targets are very similar. On the other hand, the NCLS-generated abundance fractions do not have this constraint. With this freedom, they can take whatever values that are generated by NCLS. Despite the fact that their estimated abundance fractions may not reflect accurate abundance fractions, the target detectability of NCLS may actually benefit from not satisfying the sum-to-one constraint. As a consequence, the target detection performance of NCLS is therefore enhanced. This results in better performance of NCLS than that of SCLS.

Compared to NCLS, which constrains target signature abundance fractions, CEM is a completely different approach, which constrains the desired target signature rather than its abundance fraction. It was previously developed in [12], [19], [20] for hyperspectral image classification and is not based on LSMA. It designs an adaptive filter that minimizes the filter output energy while constraining a desired target signature by a specific gain. The idea of CEM was derived from the MVDR beamformer in array processing [21], [22] and was first used in chemical remote sensing [23]. It a special case of Frost's linearly constrained adaptive beamforming approach [24]. The advantage of CEM over NCLS is that it does not require the complete knowledge of target signatures $\boldsymbol{m}_{1}, \boldsymbol{m}_{2}, \ldots, \boldsymbol{m}_{p}$ as does NCLS, but only requires the target signature to be detected, say $\boldsymbol{m}_{p}$ a priori. Using the desired target signature $\boldsymbol{m}_{p}$, one can design an adaptive filter to pass $\boldsymbol{m}_{p}$ with a specific gain while minimizing the radiance contributed by undesired signatures $\boldsymbol{m}_{1}, \boldsymbol{m}_{2}, \ldots, \boldsymbol{m}_{p-1}$, plus other unknown signal sources. Consequently, minimizing the filter output energy is equivalent to enhancing the desired target signature $\boldsymbol{m}_{p}$. CEM has been shown to be very effective in target detection for HYDICE data because it works very well for small target detection. One disadvantage of CEM is that the performance of CEM is completely determined by the signature of $\boldsymbol{m}_{p}$ as well as the true dimensionality of the data. Therefore, it is not robust and is very sensitive to $\boldsymbol{m}_{p}$ and noise. When the knowledge of the target $\boldsymbol{m}_{p}$ is not accurate or noise level is high, the performance of CEM will be significantly degraded and it may even detect wrong targets.

One common drawback of NCLS and CEM is the requirement of the prior knowledge of target signatures. For NCLS, $\boldsymbol{m}_{1}, \boldsymbol{m}_{2}, \ldots, \boldsymbol{m}_{p}$ used in LSMA must be known a priori. For CEM, knowledge of the desired signature $m_{p}$ and the intrinsic dimensionality of the data must also be known a priori. Unfortunately, finding such information in an unknown image scene is generally difficult. This is particularly true for hyperspectral imagery. For example, the HYDICE sensor has significantly improved spectral resolution to $10 \mathrm{~nm}$ and spatial resolution from 1 to $4 \mathrm{~m}$. With such fine spatial and spectral resolutions, the HYDICE sensor can extract targets with size as small as several meters for the purpose of target detection, discrimination, classification, and identification. On the other hand, many unknown interfering signatures may also be measured by the HYDICE sensor [16]. Apparently, the knowledge of such unwanted target signatures cannot be obtained a priori. In order to resolve this problem, a least squares error-based unsupervised target generation process is proposed to extend NCLS, OSP, and CEM to their unsupervised counterparts (UNCLS, UOSP, and UCEM).

The remainder of this paper is organized as follows. Section II briefly reviews the linear mixture model and unconstrained OSP method. Section III proposes a target abundance-constrained subpixel detection approach, NCLS. Section IV extends NCLS to unsupervised versions where the knowledge of target signatures is not required $a$ priori. Section $\mathrm{V}$ describes a target signature-constrained subpixel detection approach, the CEM method. Section VI conducts a series of computer simulations and hyperspectral imagery experiments to evaluate detection performance of NCLS, CEM, and OSP methods. Finally, Section VII concludes with some remarks.

\section{PRELIMINARIES: LiNEAR MiXTURE MODEL AND OSP}

\section{A. Linear Spectral Mixture Model}

Linear spectral mixing is a widely used approach for remotely sensed imagery to determine and quantify multicomponents. Since every pixel is acquired by spectral channels at different wavelengths, it can be represented by a column vector of which each component is a pixel in a particular band. More precisely, suppose that $L$ is the number of spectral bands. Let $\boldsymbol{r}$ be an $L \times 1$ column pixel vector in a multispectral or hyperspectral image where boldface is used for vectors. In this case, each multi/hyperspectral pixel is viewed as a pixel vector with $L$ dimensions. Assume that $\boldsymbol{M}$ is an $L \times p$ target spectral signature matrix denoted by $\left[\boldsymbol{m}_{1} \boldsymbol{m}_{2} \ldots \boldsymbol{m}_{p}\right]$, where $\boldsymbol{m}_{j}$ is an $L \times 1$ column vector represented by the spectral signature of the $j$ th target resident in the pixel $r$, and $p$ is the number of targets in the image scene. Let $\alpha=\left(\alpha_{1} \alpha_{2} \ldots \alpha_{p}\right)^{T}$ be a $p \times 1$ abundance column vector 
associated with $\boldsymbol{r}$, where $\alpha_{j}$ denotes the fraction of the $j$ th target signature present in the pixel vector $\boldsymbol{r}$.

A classical approach to solving mixed pixel classification problem is linear unmixing, which assumes that the spectral signatures of the $p$ endmembers in the pixel vector $\boldsymbol{r}$ are linearly mixed. Therefore, the spectral signature of a pixel vector $\boldsymbol{r}$ can be represented by a linear regression model of $\left[\boldsymbol{m}_{1} \boldsymbol{m}_{2} \ldots \boldsymbol{m}_{p}\right]$ as follows:

$$
\boldsymbol{r}=\boldsymbol{M} \alpha+\boldsymbol{n}
$$

where $\boldsymbol{n}$ is noise or can be interpreted as a measurement error.

\section{B. Orthogonal Subspace Projection (OSP)}

Equation (1) is a general linear mixture model with no constraints imposed on the abundance vector $\alpha=\left(\alpha_{1} \alpha_{2} \ldots \alpha_{p}\right)^{T}$. Recently, an unconstrained linear unmixing method, the OSP approach, was developed in [15]. It rewrites (1) as

$$
\boldsymbol{r}=\boldsymbol{d} \alpha_{p}+\boldsymbol{U} \gamma+\boldsymbol{n}
$$

where $\boldsymbol{d}=\boldsymbol{m}_{p}$ is the desired target spectral signature and $U=\left(\boldsymbol{m}_{1} \boldsymbol{m}_{2} \ldots \boldsymbol{m}_{p-1}\right)$ is the undesired target spectral signature matrix made up of the remaining $p-1$ undesired signatures in $M$. Here, without loss of generality, we assume that the last signature is the desired signature $\boldsymbol{d}$. The reason for separating $\boldsymbol{U}$ from $\boldsymbol{M}$ is that it allows us to design an orthogonal subspace projector to annihilate $\boldsymbol{U}$ from an observed pixel prior to classification. So based on (2), an OSP operator was derived in [15] by

$$
P_{\mathrm{OSP}}=\boldsymbol{d}^{T} P_{\boldsymbol{U}}^{\perp}
$$

where

$$
P_{\boldsymbol{U}}^{\perp}=I-\boldsymbol{U} \boldsymbol{U}^{\#}
$$

and $\boldsymbol{U}^{\#}=\left(\boldsymbol{U}^{T} \boldsymbol{U}\right)^{-1} \boldsymbol{U}^{T}$ is the pseudo-inverse of $\boldsymbol{U}$, and the notation $\frac{\perp}{\boldsymbol{U}}$ in $P_{\boldsymbol{U}}^{\perp}$ indicates that the projector $P_{U}^{\perp}$ maps the observed pixel $\boldsymbol{r}$ into the orthogonal complement of $\langle\boldsymbol{U}\rangle$, denoted by $\langle\boldsymbol{U}\rangle^{\perp}$.

\section{TARget Abundance-Constrained SubPIXEL DETECTION APPROACH: NONNEGATIVELY CONSTRAINED LEAST SQUARES (NCLS) METHOD}

The target signature matrix $\boldsymbol{M}$ and the abundance vector $\alpha$ in (1) are assumed to be known a priori. In reality, $\alpha$ is generally not known and needs to be estimated. In order to estimate $\alpha=$ $\left(\alpha_{1} \alpha_{2} \ldots \alpha_{p}\right)^{T}$, we use the least squares error as the criterion for optimality and the optimal least squares estimate of $\alpha, \hat{\alpha}_{\mathrm{LS}}$, for model (1) can be obtained by

$$
\hat{\alpha}_{\mathrm{LS}}=\left(\boldsymbol{M}^{T} \boldsymbol{M}\right)^{-1} \boldsymbol{M}^{T} \boldsymbol{r}
$$

As mentioned previously, the OSP classifier POSP specified by (3) does not necessarily satisfy the sum-to-one constraint $\sum_{j=1}^{p} \alpha_{j}=1$ or the nonnegativity constraint, $\alpha_{j} \geq 0$ for all $1 \leq j \leq p$. The NCLS imposes the nonnegativity constraint on the abundance vector $\alpha$ while using (5) to estimate $\alpha$. Since the nonnegativity constraint is a set of inequalities, no analytic solution can be derived to arrive at a closed form. Furthermore, since NCLS does not satisfy the sum-to-one constraint, it is not an optimal abundance estimator. So, why is it important to consider NCLS? The interesting fact is that NCLS may not be as good as an FCLS approach for endmember quantification developed in [14], but as a target detector, an NCLS-based detector may be more effective than an FCLS-based quantifier by not requiring or forcing ASC, the sum of target signature abundance fractions to one. This unconstrained disadvantage turns out to be an advantage in detection of targets for NCLS.

In general, an NCLS problem can be described by the following optimization problem

Minimize LSE $=(\boldsymbol{M} \boldsymbol{\alpha}-\boldsymbol{r})^{T}(\boldsymbol{M} \boldsymbol{\alpha}-\boldsymbol{r}) \quad$ subject to $\boldsymbol{\alpha} \geq 0$

where LSE is the least squares error used as the criterion for optimality and $\boldsymbol{\alpha} \geq 0$ represents the nonnegativity constraint $\alpha_{j} \geq 0$ for all $1 \leq \bar{j} \leq p$. Since $\boldsymbol{\alpha} \geq 0$ is a set of inequalities, the Lagrange multiplier method is not applicable to solving optimal solutions. In order to mitigate this dilemma, we introduce an unknown $p$-dimensional positive constraint constant vector $\boldsymbol{c}=$ $\left[c_{1} c_{2} \ldots c_{p}\right]^{T}>0$ with $c_{j}>0$ for $1 \leq j \leq p$ to take care of the nonnegativity constraint. Through $c$, we can form a Lagrangian $J$ as follows:

$$
J=\frac{1}{2}(M \alpha-\boldsymbol{r})^{T}(M \alpha-\boldsymbol{r})+\lambda(\alpha-\boldsymbol{c})
$$

with $\alpha=\boldsymbol{c}$ and

$$
\left.\frac{\partial J}{\partial \alpha}\right|_{\hat{\alpha}_{\mathrm{NCLS}}}=0 \Rightarrow \boldsymbol{M}^{T} \boldsymbol{M} \hat{\alpha}_{\mathrm{NCLS}}-M^{T} \boldsymbol{r}+\lambda=0
$$

which results in the following two iterative equations given by

$$
\begin{aligned}
\hat{\alpha}_{\mathrm{NLCS}} & =\left(\boldsymbol{M}^{T} \boldsymbol{M}\right)^{-1} \boldsymbol{M}^{T} \boldsymbol{r}-\left(\boldsymbol{M}^{T} \boldsymbol{M}\right)^{-1} \lambda \\
& =\hat{\alpha}_{\mathrm{LS}}-\left(\boldsymbol{M}^{T} \boldsymbol{M}\right)^{-1} \lambda
\end{aligned}
$$

and

$$
\lambda=\boldsymbol{M}^{T}\left(\boldsymbol{r}-\boldsymbol{M} \hat{\alpha}_{\mathrm{NLCS}}\right) .
$$

Equations (9) and (10) can be used to solve the optimal solution $\hat{\alpha}_{\mathrm{NLCS}}$ and the Lagrange multiplier vector $\lambda=\left(\lambda_{1} \lambda_{2} \ldots \lambda_{p}\right)^{T}$.

The nonnegativity constraint optimization problem given by (6) was previously explored by Lawson and Hanson in [17] and was called the NNLS. Based on Lawson and Hanson's NNLS, two Fast NNLS algorithms referred to as FNNLS and FNNLSb were further developed by Bro and Jong in [18] to generate the desired optimal solutions. Their idea is to first decompose the components of the estimate $\hat{\alpha}_{\mathrm{LS}}$ into two index sets called active set and passive set. While the former consists of all indices corresponding to negative (or zero) components in the estimate $\hat{\alpha}_{\mathrm{LS}}$, the latter contains all indices corresponding to positive components in the estimate $\hat{\alpha}_{\mathrm{LS}}$. NNLS and FNNLS start off with an empty passive set $P=\emptyset$ and assume the active set consisting all components of $\hat{\alpha}_{\mathrm{LS}}$, i.e., $R=\{1,2, \ldots, p\}$. They then adjust both sets $P$ and $R$ via iterations using (10). It 
has been shown in [17] that when an optimal solution has been found, the Lagrange multiplier vector $\lambda$ must satisfy the following Kuhn-Tucker conditions:

$$
\begin{array}{ll}
\lambda_{j}=0, & j \in P \\
\lambda_{j}<0, & j \in R .
\end{array}
$$

The final generated passive set identifies which components are legitimate to be used in the abundance estimation $\hat{\alpha}_{\mathrm{LS}}$. The details of implementing the NCLS algorithm are given below.

\section{NCLS Algorithm}

1) Initialization: Set the passive set $P^{(0)}=\{1,2, \ldots p\}$ and active set $R^{(0)}=\emptyset$. Set $k=0$

2) Compute $\hat{\alpha}_{\mathrm{LS}}$ using (5). Let $\hat{\alpha}_{\mathrm{NLCS}}^{(k)}=\hat{\alpha}_{\mathrm{LS}}$.

3) At the $k$ th iteration. If all components in $\hat{\alpha}_{\text {NLCS }}^{(k)}$ are nonnegative, the algorithm is terminated. Otherwise, continue.

4) Let $k=k+1$

5) Move all indices in $P^{(k-1)}$ that correspond to negative components of $\hat{\alpha}_{\mathrm{NLCS}}^{(k-1)}$ to $R^{(k-1)}$, and the resulting index sets are denoted by $P^{(k)}$ and $R^{(k)}$, respectively. Create a new index set $S^{(k)}$ and set it equal to $R^{(k)}$

6) Let $\hat{\alpha}_{R^{(k)}}$ denote the vector consisting of all components $\hat{\alpha}_{\mathrm{LS}}$ in $R^{(k)}$

7) Form a steering matrix $\Phi_{\alpha}^{(k)}$ by deleting all rows and columns in the matrix $\left(\boldsymbol{M}^{T} \boldsymbol{M}\right)^{-1}$ that are specified by $P^{(k)}$

8) Calculate $\lambda^{(k)}=\left(\Phi_{\alpha}^{(k)}\right)^{-1} \hat{\alpha}_{R^{(k)}}$. If all components in $\lambda^{(k)}$ are negative, go to step 13. Otherwise, continue.

9) Calculate $\lambda_{\max }^{(k)}=\arg \left\{\max _{j} \lambda_{j}^{(k)}\right\}$ and move the index in $R^{(k)}$ that corresponds to $\lambda_{\max }^{(k)}$ to $P^{(k)}$.

10) Form another matrix $\Psi_{\lambda}^{(k)}$ by deleting every column of $\left(\boldsymbol{M}^{T} \boldsymbol{M}\right)^{-1}$ specified by $P^{(k)}$.

11) Set $\hat{\alpha}_{S^{(k)}}=\hat{\alpha}_{\mathrm{LS}}-\Psi_{\lambda}^{(k)} \lambda^{(k)}$.

12) If any components of $\hat{\alpha}_{S^{(k)}}$ in $S^{(k)}$ are negative, then move these components from $P^{(k)}$ to $R^{(k)}$. Go to step 6 .

13) Form another matrix $\Psi_{\lambda}^{(k)}$ by deleting every column of $\left(\boldsymbol{M}^{T} \boldsymbol{M}\right)^{-1}$ specified by $P^{(k)}$.

14) Set $\hat{\alpha}_{\text {NLCS }}^{(k)}=\hat{\alpha}_{\mathrm{LS}}-\Psi_{\lambda}^{(k)} \lambda^{(k)}$. Go to step 3 .

In summary, at the $k$ th iteration, the NCLS algorithm begins by calculating the unconstrained least squares solution $\hat{\alpha}_{\mathrm{LS}}$. If all components in $\hat{\alpha}_{\mathrm{LS}}$ are positive, the algorithm terminates. Otherwise, all negative components are identified, and their corresponding indices are moved to the active set $R^{(k)}$. In the meantime, a duplicate set of $R^{(k)}$, referred to as $S^{(k)}$, is introduced for the purpose of keeping track of the current negative components of $\hat{\alpha}_{\mathrm{NLCS}}^{(k-1)}$ during the $k$ th iteration. The steering matrix $\Phi_{\alpha}^{(k)}$ is then formed and the Lagrange multiplier vector $\lambda^{(k)}$ that will be used to steer each negative component of $\hat{\alpha}_{\mathrm{NLCS}}^{(k-1)}$ back to zero is calculated. From (11), all components of $\lambda^{(k)}$ must be negative. Therefore, in case there exists at least one positive component, the index that corresponds to the maximum component of $\lambda^{(k)}$ is shuffled from $R^{(k)}$ to $P^{(k)}$. Since the loop from step 6 to step 12 may be repeated over and over again during a single iteration, $S^{(k)}$ is used to check if all previously identified indices of maximum components of $\lambda^{(k)}$ should be retained in $P^{(k)}$ or moved back to $R^{(k)}$. Once all the values of $\lambda^{(k)}$ are negative, $\hat{\alpha}_{\text {NLCS }}^{(k)}$ is recalculated. It should be noted that the two iterative equations, specified by (9) and (10), are carried out by step 14 and step 8 respectively.

One comment on NCLS is noteworthy. The reason that people overlook the potential application of NCLS in subpixel target detection is because it requires the complete prior knowledge of targets present in an image where NCLS is primarily used for unmixing materials rather than detecting a particular target in a linear mixture. Of course, in this case, NCLS cannot compete with the FCLS in [14].

\section{UNSUPERVISED NCLS}

NCLS requires the complete knowledge of target signatures. When no prior information is available, it cannot be applied. In this section, the NCLS is extended to an unsupervised version where the LSE is used to minimize the goodness of fit between the linear mixture model and data measurements. The idea can be briefly described as follows.

Initially, we can select any arbitrary pixel vector as an initial desired target signature denoted by $\boldsymbol{t}_{0}$. However, a good choice may be the pixel vector, with a maximum length that corresponds to the brightest pixel in the image scene. The NCLS algorithm is then used to estimate the abundance fraction of $t_{0}$, denoted by $\hat{\alpha}_{0}^{(1)}(\boldsymbol{r})$ for each pixel vector $\boldsymbol{r}$ in the scene, and the LSE is further calculated between the image pixel vector $\boldsymbol{r}$ and its estimate $\hat{\alpha}_{0}^{(1)}(\boldsymbol{r}) \boldsymbol{t}_{0}$, i.e.

$$
\operatorname{LSE}^{(0)}(\boldsymbol{r})=\left[\left(\hat{\alpha}_{0}^{(1)}(\boldsymbol{r}) \boldsymbol{t}_{0}-\boldsymbol{r}\right)^{T}\left(\hat{\alpha}_{0}^{(1)}(\boldsymbol{r}) \boldsymbol{t}_{0}-\boldsymbol{r}\right)\right] .
$$

Here, $\boldsymbol{r}$ is included in the abundance fraction estimate $\hat{\alpha}_{0}^{(1)}(\boldsymbol{r})$ to emphasize that the estimated abundance fraction $\hat{\alpha}_{0}$ is a function of the pixel vector $\boldsymbol{r}$ and varies with $\boldsymbol{r}$. The pixel that yields the maximum LSE is then selected as the next target signature, denoted by $\boldsymbol{t}_{1}$, namely

$$
\begin{aligned}
\max _{\boldsymbol{r}} & \operatorname{LSE}^{(0)}(\boldsymbol{r}) \\
& =\operatorname{LSE}^{(0)}\left(\boldsymbol{t}_{1}\right) \\
& =\left[\left(\hat{\alpha}_{0}^{(1)}\left(\boldsymbol{t}_{1}\right) \boldsymbol{t}_{0}-\boldsymbol{t}_{1}\right)^{T}\left(\hat{\alpha}_{0}^{(1)}\left(\boldsymbol{t}_{1}\right) \boldsymbol{t}_{0}-\boldsymbol{t}_{1}\right)\right] .
\end{aligned}
$$

Because the LSE between $\hat{\alpha}_{0}^{(1)}\left(\boldsymbol{t}_{1}\right) \boldsymbol{t}_{0}$ and $\boldsymbol{t}_{1}$ is the maximum, it can be expected that $t_{1}$ is most dissimilar to $t_{0}$. In order to find a second target signature, the UNCLS algorithm estimates the abundance fractions of $t_{0}$ and $t_{1}$ contained in each pixel vector $\boldsymbol{r}$ in the image scene, denoted by $\hat{\alpha}_{0}^{(2)}(\boldsymbol{r})$ and $\hat{\alpha}_{1}^{(2)}(\boldsymbol{r})$. Then the maximum LSE between all image pixel vectors $r$ and the least squares linear mixture $\hat{\alpha}_{0}^{(2)}(\boldsymbol{r}) \boldsymbol{t}_{0}+\hat{\alpha}_{1}^{(2)}(\boldsymbol{r}) \boldsymbol{t}_{1}$ are estimated by the NCLS algorithm. Once again, the pixel vector that yields the maximum LSE is selected as a second target signature denoted by $t_{2}$. The same procedure of using the NCLS algorithm with $m=\left[t_{0} t_{1} t_{2}\right]$ is repeated until the resulting LSE is small enough and less than a prescribed error threshold. It should be noted that if there is partial knowledge available $a$ priori, it can be incorporated in the above process. For example, if we know nothing but the desired target signature $\boldsymbol{d}$, the initial target pixel vector $\boldsymbol{t}_{0}$ can be replaced by this $\boldsymbol{d}$. If there is more than one 
known target in the image scene, we can select these targets as an initial target set and then follow the same procedure described above until the LSE meets a stopping criterion. The procedure outlined as above is called the UNCLS algorithm, which can be summarized as follows.

\section{Unsupervised NCLS (UNCLS) Algorithm}

1) Initial condition: Select $\varepsilon$ to be a prescribed error threshold and let $\boldsymbol{t}_{0}=\arg \left\{\max _{\boldsymbol{r}}\left[\boldsymbol{r}^{T} \boldsymbol{r}\right]\right\}$ where $\boldsymbol{r}$ is run over all image pixel vectors. Let $k=0$.

2) Let $k \leftarrow k+1$, and apply the NCLS algorithm with the signature matrix $\boldsymbol{M}=\left[\boldsymbol{t}_{0} t_{1} \ldots \boldsymbol{t}_{k-1}\right]$ to estimate the abundance fractions of $\boldsymbol{t}_{0}, \boldsymbol{t}_{1}, \ldots, \boldsymbol{t}_{k-1}, \hat{\alpha}_{0}^{(k)}(\boldsymbol{r})$, $\hat{\alpha}_{1}^{(k)}(\boldsymbol{r}), \ldots, \hat{\alpha}_{k-1}^{(k)}(\boldsymbol{r})$.

3) Find the LSE defined by

$$
\begin{aligned}
\operatorname{LSE}^{(k-1)}(\boldsymbol{r})= & \left(\boldsymbol{r}-\left[\sum_{i=0}^{k-1} \hat{\alpha}_{i}^{(k)}(\boldsymbol{r}) \boldsymbol{t}_{i}\right]\right)^{T} \\
& \cdot\left(\boldsymbol{r}-\left[\sum_{i=0}^{k-1} \hat{\alpha}_{i}^{(k)}(\boldsymbol{r}) \boldsymbol{t}_{i}\right]\right)
\end{aligned}
$$

and check the error if $\operatorname{LSE}^{(k-1)}(\boldsymbol{r})<\varepsilon$ for all $\boldsymbol{r}$. If it is, the algorithm stops, otherwise continue.

4) Find $\boldsymbol{t}_{k}=\arg \left\{\max \boldsymbol{r} \operatorname{LSE}^{(k-1)}(\boldsymbol{r})\right\}$. Go to step 2 .

One comment on $\hat{\alpha}_{j}^{(k)}(\boldsymbol{r})$ is noteworthy. The superscript $(k)$ is a counter to indicate the number of iterations. It starts with $k=1$. The subscript $j$ starting with $j=1$ is the index of the $j$ th target signature $\boldsymbol{t}_{j}$, generated by the UNCLS algorithm. The initial target is represented by $\boldsymbol{t}_{0}$ with $j=0$. For example, $\hat{\alpha}_{0}^{(1)}(\boldsymbol{r})$ is the abundance estimate of $\boldsymbol{t}_{0}$ in the first iteration, given by (13). It should also be noted that, as will be demonstrated in the experiments in Section VI, step 4 implemented in the UNCLS algorithm tries to locate pure pixel vectors first. If there is no such pixel vector, it then looks for a mixed pixel vector with the largest possible abundance fraction of any substance in the pixel vector. This implies that a mixed pixel vector with uniform mixture is less likely to be selected by the UNCLS as a target signature. Furthermore, using an analogous approach to UNCLS, OSP and CEM can be also implemented in an unsupervised fashion, referred to as UOSP and UCEM throughout this paper.

\section{TARget Signature-Constrained SubPiXel Detection APPROACH: CEM}

In order to implement the NCLS algorithm, knowledge of all target signatures of interest is required. Such knowledge is generally difficult to obtain in practice. So, a least squares error-based unsupervised method, as described in Section III, was proposed for this purpose. As an alternative, the CEM approach [12], [19], [20] recently proposed in [12] took another approach. Instead of constraining target signature abundance fractions, CEM constrains a desired target signature by using a specific gain. Since CEM does not impose a constraint on the abundance of the target signature, it cannot be used for quantification purposes as the FCLS in [14]. However, as a target detector, CEM has shown success in hyperspectral image classification [19], [20].

Basically, CEM uses an FIR filter to constrain the desired target signature by a specific gain while minimizing the filter output power. The idea of CEM arises from the MVDR in array processing [21], [22], with the desired target signature interpreted as the direction of arrival from a desired signal. It can be derived as follows.

Assume that we are given a finite set of observations $S=$ $\left\{\boldsymbol{r}_{1} \boldsymbol{r}_{2} \ldots \boldsymbol{r}_{N}\right\}$ where $\boldsymbol{r}_{i}=\left(r_{i 1} r_{i 2} \ldots r_{i L}\right)^{T}$ for $1 \leq i \leq N$ is a sample pixel vector. Suppose that the desired target signature $\boldsymbol{d}$ is also known a priori. The objective of CEM is to design an FIR linear filter with $L$ filter coefficients $\left\{w_{1} w_{2} \ldots w_{L}\right\}$ denoted by an $L$-dimensional vector $\boldsymbol{w}=\left(w_{1} w_{2} \ldots w_{L}\right)^{T}$ that minimizes the filter output power subject to the following constraint

$$
\boldsymbol{d}^{T} \boldsymbol{w}=\sum_{l=1}^{L} d_{l} w_{l}=1 .
$$

It is worth noting that the constraint constant 1 in (15) can be replaced by any scalar $\boldsymbol{c}$ [21], [22].

Let $y_{i}$ denote the output of the designed FIR filter resulting from the input $\boldsymbol{r}_{i}$. Then $y_{i}$ can be written as

$$
y_{i}=\sum_{l=1}^{L} w_{l} r_{i l}=\boldsymbol{w}^{T} \boldsymbol{r}_{i}=\boldsymbol{r}_{i}^{T} \boldsymbol{w} .
$$

So the average output power produced by the observation set $S$ and the FIR filter with coefficient vector $\boldsymbol{w}=\left(w_{1} w_{2} \ldots w_{L}\right)^{T}$ specified by (16) is given by

$$
\begin{aligned}
\frac{1}{N}\left[\sum_{i=1}^{N} y_{i}^{2}\right] & =\frac{1}{N}\left[\sum_{l=1}^{L}\left(\boldsymbol{r}_{i}^{T} \boldsymbol{w}\right)^{T} \boldsymbol{r}_{i}^{T} \boldsymbol{w}\right] \\
& =\boldsymbol{w}^{T}\left(\frac{1}{N}\left[\sum_{i=1}^{N} \boldsymbol{r}_{i} \boldsymbol{r}_{i}^{T}\right]\right) \boldsymbol{w} \\
& =\boldsymbol{w}^{T} R_{L \times L} \boldsymbol{w}
\end{aligned}
$$

where $R_{L \times L}=1 / N\left[\sum_{i=1}^{N} \boldsymbol{r}_{i} \boldsymbol{r}_{i}^{T}\right]$ turns out to be the $L \times L$ sample autocorrelation matrix of $S$. Minimizing (17) with the filter response constraint $\boldsymbol{d}^{T} \boldsymbol{w}=\sum_{l=1}^{L} d_{l} w_{l}=1$ yields

$$
\begin{aligned}
\min _{\boldsymbol{w}}\left\{\frac{1}{N}\left[\sum_{i=1}^{N} y_{i}^{2}\right]\right\}=\min _{\boldsymbol{w}}\left\{\boldsymbol{w}^{T} R_{L \times L} \boldsymbol{w}\right\} \\
\text { subject to } \boldsymbol{d}^{T} \boldsymbol{w}=1 .
\end{aligned}
$$

The solution to (18) was shown in [12] and called the CEM filter with the weight vector $\boldsymbol{w}^{*}$ given by

$$
\boldsymbol{w}^{*}=\frac{R_{L \times L}^{-1} \boldsymbol{d}}{\boldsymbol{d}^{T} R_{L \times L}^{-1} \boldsymbol{d}} .
$$

It should be noted that the $R_{L \times L}$ in (19) is not necessarily of full rank. So calculating the $R_{L \times L}^{-1}$ in (19) can be a problem. It has been noted that CEM is very sensitive to the knowledge used for the desired target as well as the noise. While the problem of CEM's sensitivity to the target signature knowledge has been addressed in [25], the issue of CEM's noise sensitivity has not 
been studied in depth in open literature. As will also be shown in the experiments, the noise sensitivity is closely related to the rank used to calculate the weight vector in (19). This rank determines the number of eigenvectors to be used to calculate $R_{L \times L}^{-1}$ and is also closely related to the intrinsic dimensionality of a hyperspectral image, which is usually less than the data dimensionality $L$. It is known that finding the intrinsic dimensionality of data is very challenging and has been investigated previously in [26], [27]. Nevertheless, if the number of eigenvectors is known a priori ( $q$, for example), we can use singular value decomposition so that $R_{L \times L}$ can be reduced to $\tilde{R}_{L \times L}=\tilde{V} \Lambda \tilde{V}^{T}$, where $\tilde{V}=\left(\tilde{\boldsymbol{v}}_{1} \tilde{\boldsymbol{v}}_{2} \ldots \tilde{\boldsymbol{v}}_{q}\right)$ is an eigenmatrix, $\tilde{v}_{k}$ is the $L$-dimensional vector corresponding to the $k$ th eigenvalue $\lambda_{k}$, and $\Lambda=\operatorname{diag}\left\{\lambda_{1}, \lambda_{2}, \ldots, \lambda_{q}\right\}$ is a diagonal matrix with eigenvalues as diagonal elements. Using this eigen-decomposition, the inverse of $\tilde{R}_{L \times L}=\tilde{V} \Lambda \tilde{V}^{T}$ can be found by $\tilde{R}_{L \times L}^{-1}=\tilde{V} \Lambda^{-1} \tilde{V}^{T}[12]$.

\section{COMPUTER SimULATIONS AND EXPERIMENTS}

In this section, a series of computer simulations and real hyperspectral data experiments are conducted to evaluate the comparative performance among the three subpixel target detection techniques, unconstrained OSP, target abundance-constrained NCLS, and target signature-constrained CEM methods along with their unsupervised counterparts. In particular, a comprehensive analysis on the issues of noise sensitivity and target knowledge is studied by simulating various scenarios to see the effects of different numbers of eigenvectors $q$ used in computation of $R_{L \times L}^{-1}$ on the performance of CEM, as well as the impacts of different levels of prior target signature knowledge used in NCLS, OSP, and CEM

\section{A. Computer Simulations}

Three examples are designed to demonstrate two important issues of subpixel detection: noise sensitivity and sensitivity to prior target knowledge.

1) Noise Sensitivity to Number of Eigenvectors Used in Computation of $R_{L \times L}^{-1}$ : Determining the number of eigenvectors $q$ is always challenging because it is closely related to the unpredictable noise level incurred in the data. However, except for Gaussian noise, it is generally difficult to simulate non-Gaussian random noise. So in the following experiments, instead of directly considering such random noise, we deal with the issue of the effects caused by $q$ since the selection of $q$ is determined by the noise sensitivity.

Example 1: Target Signatures with Relatively Large Abundance Fractions: A laboratory data set of an AVIRIS scene considered in [15] was used to evaluate the performance of NCLS and CEM against OSP. The data set contained five field reflectance spectra, dry grass, red soil, creosote leaves, blackbrush, and sagebrush shown in Fig. 1(a) with spectral range from $0.4-2.5 \mu \mathrm{m}$. There were 158 bands after water bands, and bands with low SNR were removed. In this case, the signature matrix was $\boldsymbol{M}=\left[\boldsymbol{m}_{1} \boldsymbol{m}_{2} \boldsymbol{m}_{3} \boldsymbol{m}_{4} \boldsymbol{m}_{5}\right]$ consisting of these five spectral signatures with abundance fractions given by $\alpha=\left(\alpha_{1} \alpha_{2} \alpha_{3} \alpha_{4} \alpha_{5}\right)^{T}$. The simulation consisted of 400 mixed pixel vectors and was divided into four groups, each of which contained a hundred pixel vectors with the same mixture. The first group consisted of the first hundred pixel vectors with the mixture made up of 50\% sagebrush and 50\% dry grass. The second group consisted of the second hundred pixel vectors with the mixture made up of 50\% sagebrush and $50 \%$ red soil. The third group consisted of the third hundred pixel vectors with the mixture made up of $50 \%$ sagebrush and $50 \%$ creosote leaves. The fourth group consisted of the fourth hundred pixel vectors with the mixture made up of $50 \%$ sagebrush and 50\% blackbrush. More specifically, each of the 400 simulated pixel vectors is a two-component mixture with a 50/50 split, and all of the 400 pixel vectors share the same amount of sagebrush (that is, 50\% sagebrush). White Gaussian noise was added to each pixel vector to achieve a 30:1 SNR, which was defined in [15] as 50\% reflectance divided by the standard deviation of the noise. Fig. 1(b)-(f) shows the results of NCLS, CEM, and OSP in detection of dry grass, red soil, creosote leaves, blackbrush, and sagebrush, respectively. From Fig. 1, both OSP and NCLS were able to detect all of the five signatures. However, if we examine the amounts of abundance detected by NCLS and OSP, those detected by OSP did not reflect true abundance fractions, but those produced by NCLS did. This is because OSP is unconstrained and NCLS is at least partially constrained. Surprisingly, CEM, which was shown to be effective in [12], [19], and [20], performed poorly. As noted in the concluding remark of Section IV, the number of eigenvectors (denoted by $q$ ) used to calculate $R_{L \times L}^{-1}$ in (19) is crucial. Since there are five signatures in the simulated data, $q$ was chosen to be 5 to produce the results in Fig. 1. In order to see if $q=5$ was appropriate, $3,10,60$, and 158 were also tested for $q$ to detect blackbrush, and the results are shown in Fig. 2. As we can see, at $q=3$, the mixture of creosote leaves and sagebrush resulted in a large value above 1 instead of the mixture of blackbrush and sagebrush. The value $q=5$ yielded the best result, and as $q$ increased past 5 , the results became worse. In the case of this experiment, $q=5$ was the optimal number of eigenvectors to be used in computation of $R_{L \times L}^{-1}$.

Example 2: Target Signatures with Small Amount of Abundance Fractions: The experiment conducted in this example provides another extreme for CEM performance. The data to be used are three field reflectance spectra, dry grass, red soil, and creosote leaves shown in Fig. 1(a). Again, 400 mixed pixel vectors were also generated, but simulated in a different way from that in Example 1. We started the first pixel vector with $100 \%$ red soil and $0 \%$ dry grass, then began to increase $0.25 \%$ dry grass and decrease $0.25 \%$ red soil every pixel vector until the 400th pixel vector, which contained $100 \%$ dry grass. We then added creosote leaves to pixel vector numbers 198-202 at abundance fractions $10 \%$, while reducing the abundance of red soil and dry grass accordingly. For example, after addition of creosote leaves, the resulting pixel vector 200 contained $10 \%$ creosote leaves, $45 \%$ red soil, and $45 \%$ dry grass. White Gaussian noise was also added to each pixel vector to achieve a $30: 1$ SNR. Fig. 3 shows the results of OSP, CEM and NCLS in detection of creosote leaves. Unlike Example 1, this time all three methods NCLS, CEM, and OSP produced comparable detection results. However, both NCLS and CEM performed better than OSP in the sense of detecting true abundance fractions of 


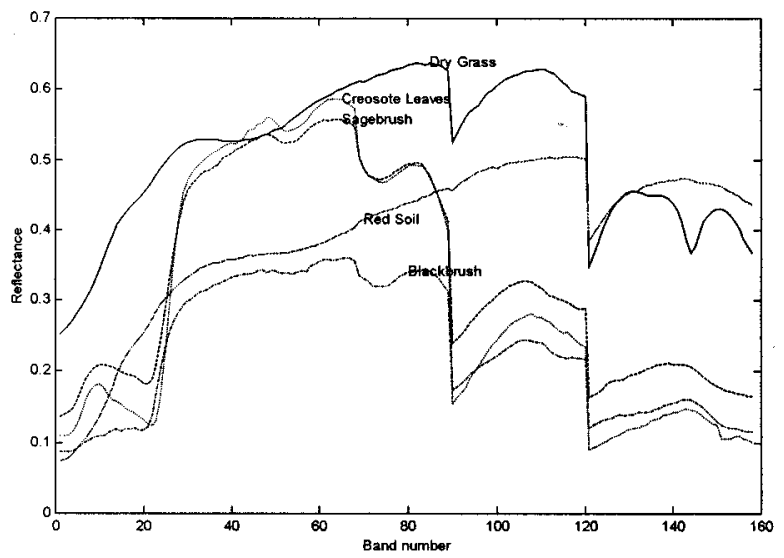

(a)
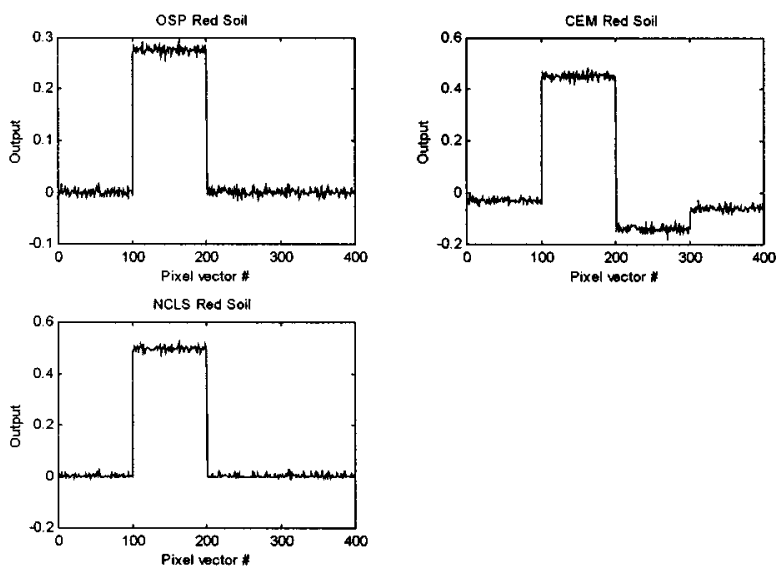

(c)
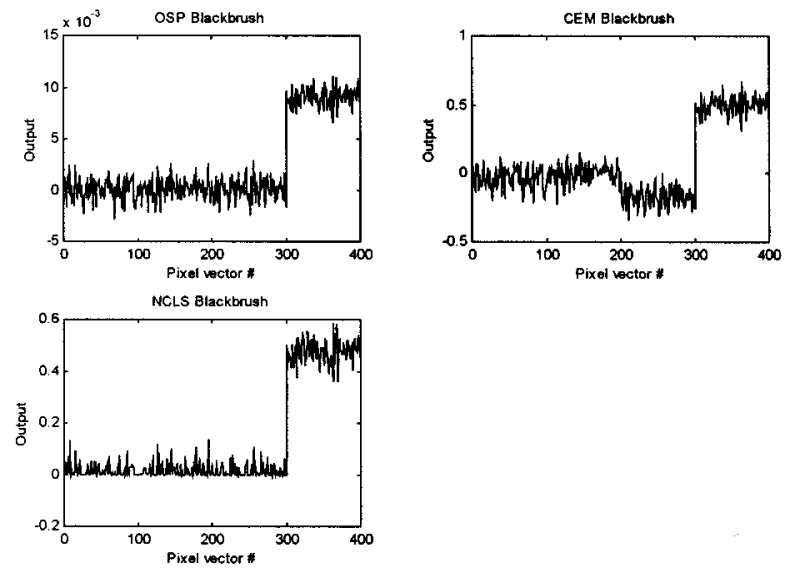

(e)
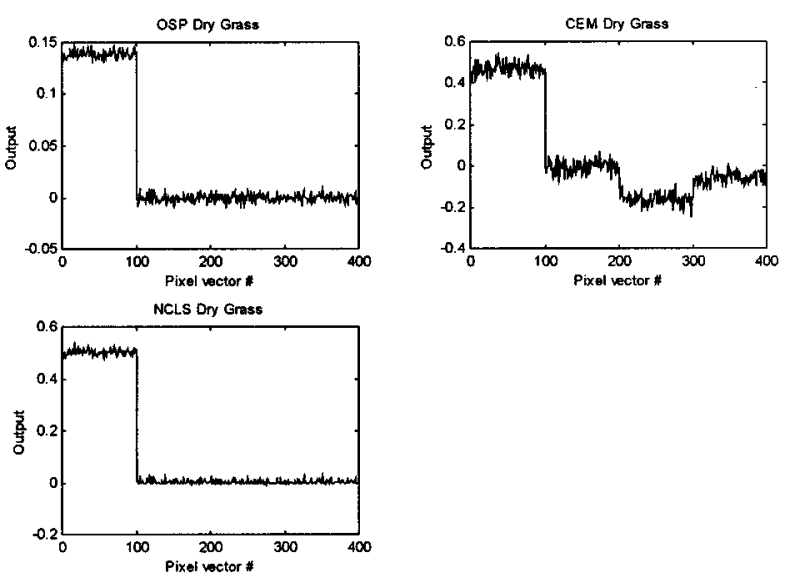

(b)
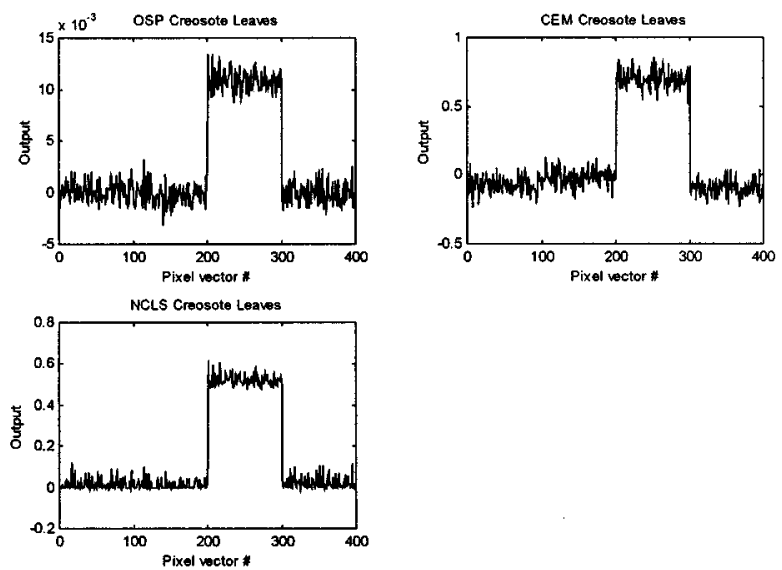

(d)
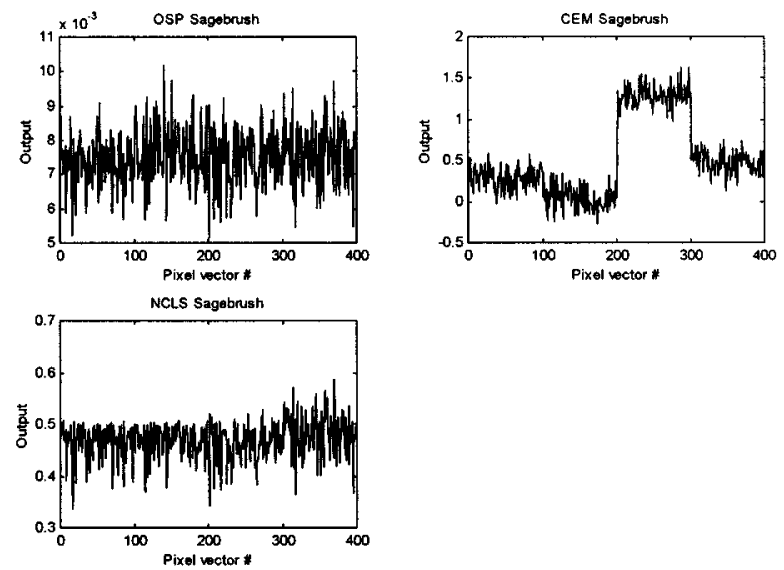

(f)

Fig. 1. (a) Refectances of five signatures, dry grass, red soil, creosote leaves, blackbrush, and sagebrush. (b)-(f) Results of OSP, CEM, and NCLS in detection of dry grass, red soil, creosote leaves, blackbrush, and sagebrush.

creosote leaves, whereas OSP did not. Compared to $q=5$, used to produce Fig. 1(d), the number of eigenvectors $q$ used to calculate $R_{L \times L}^{-1}$ in Fig. 3 was set to the full dimensionality of pixel vector 158. Fig. 4 shows the results of CEM using the number of eigenvectors $q=2,3,10,60$. Interestingly, when $q=2$, CEM picked up a wrong target signature: dry grass which was supposed to be creosote leaves [see Fig. 4(a)]. For CEM, $q=60$ yielded the best result, shown in Fig. 4(d), where the estimated abundance of creosote leaves was nearly accurate. But even in this case, the result was still not as good as that produced by NCLS in Fig. 3(c), because of the performance in detecting abundance fractions of other pixels.

While Example 1 shows one extreme case for CEM performance, Example 2 provides another extreme case for CEM. Both 

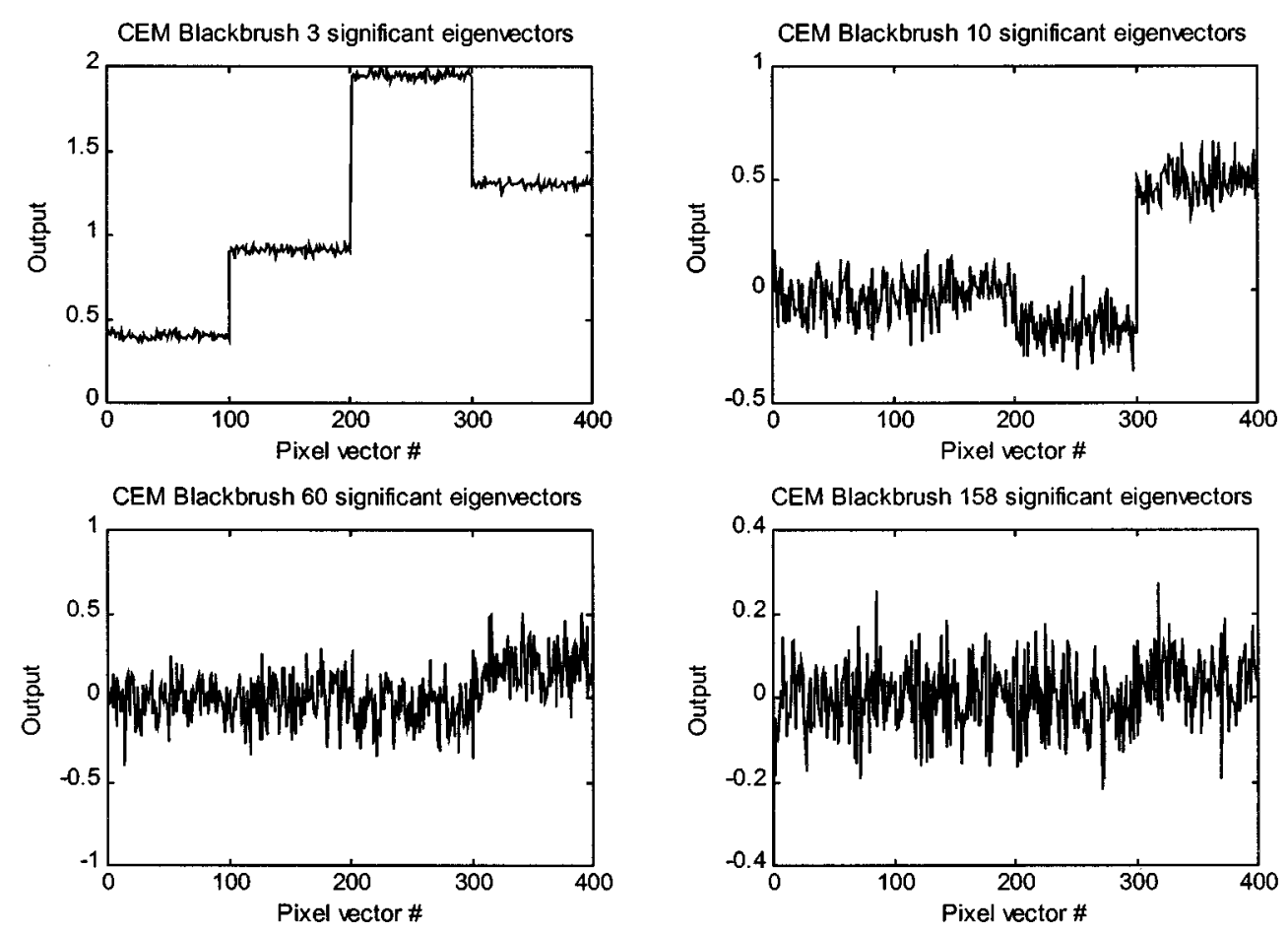

Fig. 2. Results of CEM using the number of eigenvectors $q=3,10,60$, and 158 with blackbrush as the desired signature.

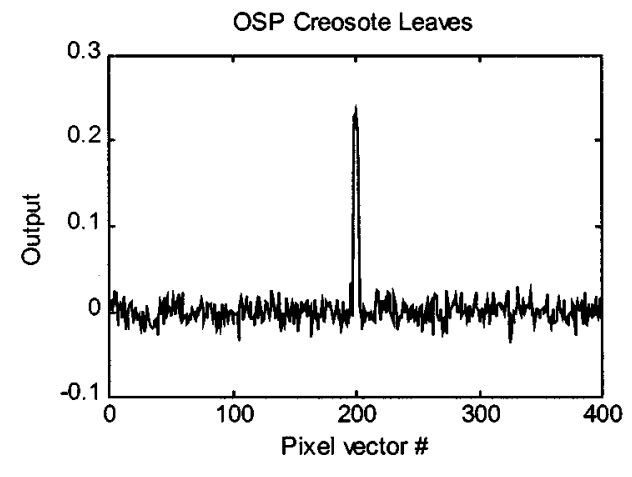

NCLS Creosote Leaves

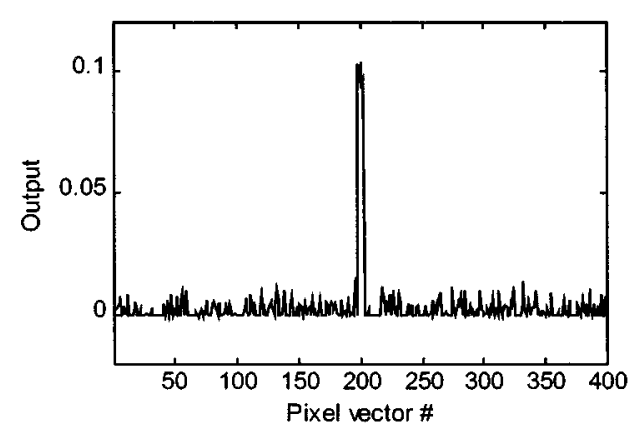

Fig. 3. Results of OSP, CEM, and NCLS in detection of creosote leaves.

examples further demonstrate the crucial role the number of eigenvectors $q$ plays in CEM performance. If each eigenvector is interpreted as a piece of information, the larger the eigenvalue is, the more significant information it represents. So these two examples suggest that when the desired target is small or occurs with low probability, the number of eigenvectors to be used for $q$ is generally very high because targets with small abundance

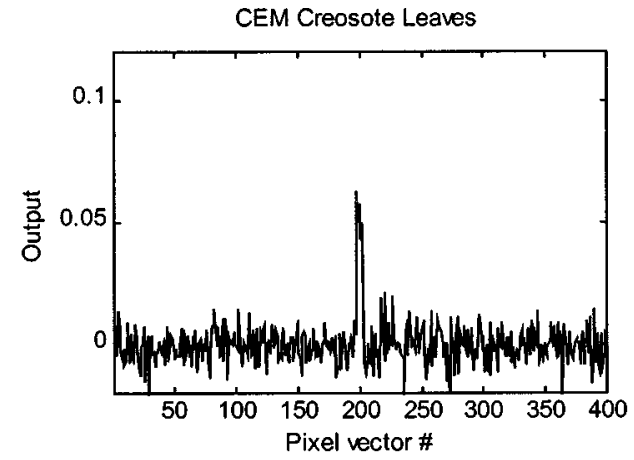

fractions may correspond to small eigenvalues and can be then viewed as insignificant targets. Under this circumstance, they may not be able to be detected by using only a few eigenvectors. Therefore, it requires a large set of eigenvectors to find these targets. This explains why CEM can be used to detect small targets so effectively by letting $q$ be equal to the full number of full bands. Conversely, if the desired targets are relatively large 

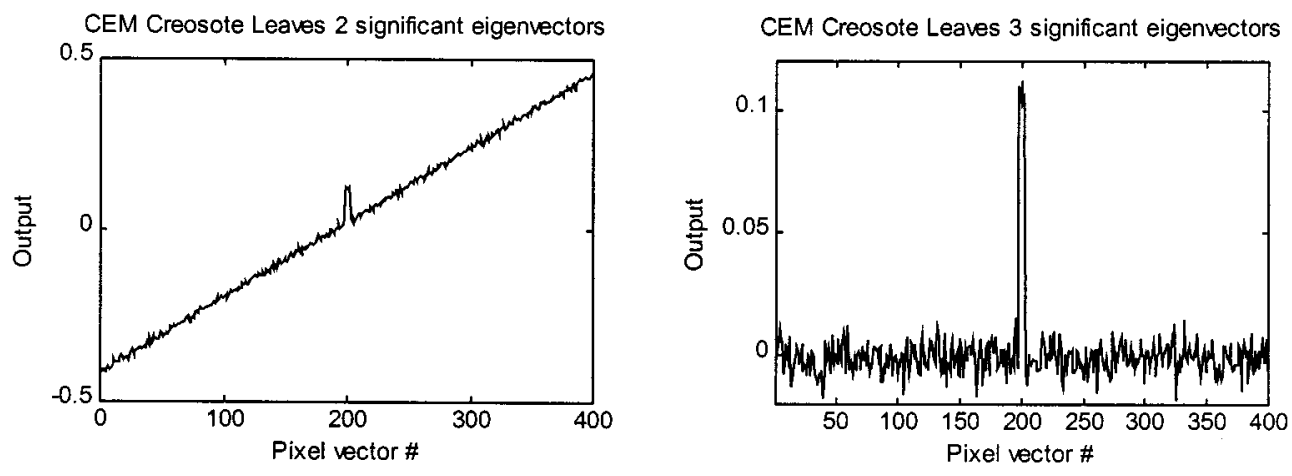

CEM Creosote Leaves 10 significant eigenvectors

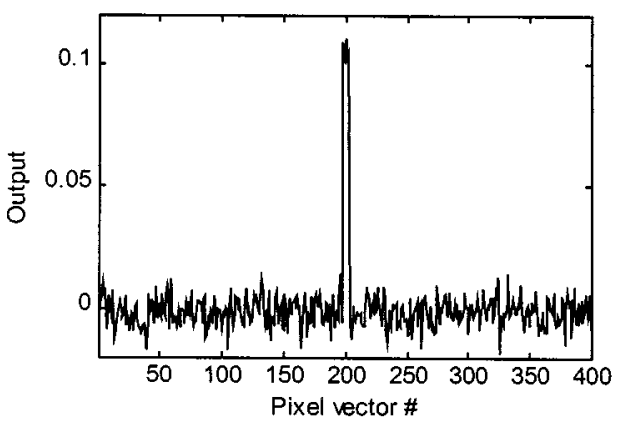

CEM Creosote Leaves 60 significant eigenvectors

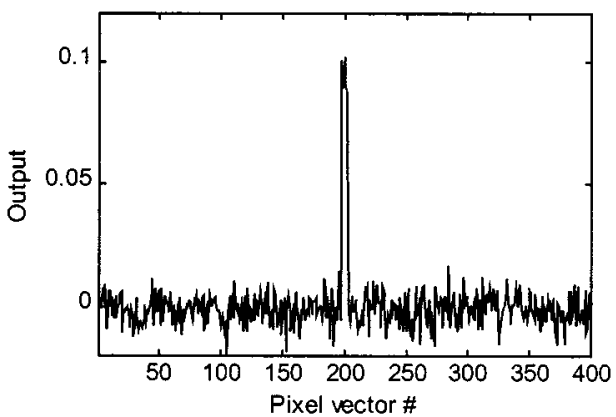

Fig. 4. Results of CEM using the number of eigenvectors $q=2,3,10,60$ in the detection of creosote leaves.
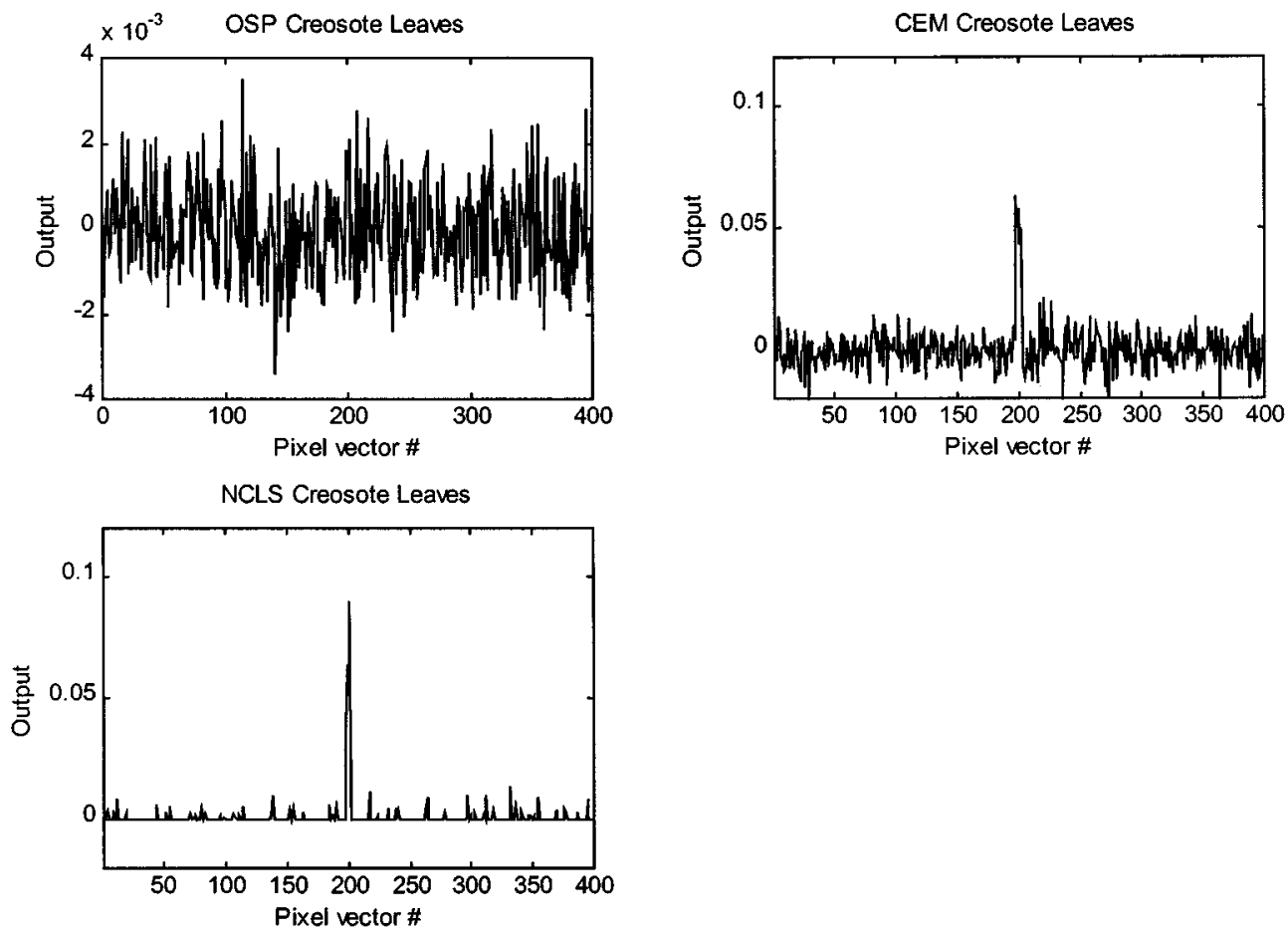

Fig. 5. Results of NCLS, CEM, and OSP in detection of creosote leaves with blackbrush and sagebrush acted as interferers.

and widespread, like the one studied in Example 1, a smaller $q$ may be more appropriate to make CEM effective because the information provided by these targets can be well-represented by a few largest eigenvectors. In this case, a small set of large eigenvectors may be sufficient to detect these targets.

Example 3: Target Signatures Used as Interferers: The same simulated data used in Example 2 were also used in this example, except that two more additional signatures, blackbrush and sagebrush, were assumed to be present in the data even though they were actually not present. In this case, the signature matrix $\boldsymbol{M}=\left[\boldsymbol{m}_{1} \boldsymbol{m}_{2} \boldsymbol{m}_{3} \boldsymbol{m}_{4} \boldsymbol{m}_{5}\right]$ contained the five signatures: dry grass, red soil, creosote leaves, blackbrush, and sagebrush in Fig. 1. With this scenario, the blackbrush and sagebrush acted as interferers rather than target signatures. Fig. 5 shows the re- 

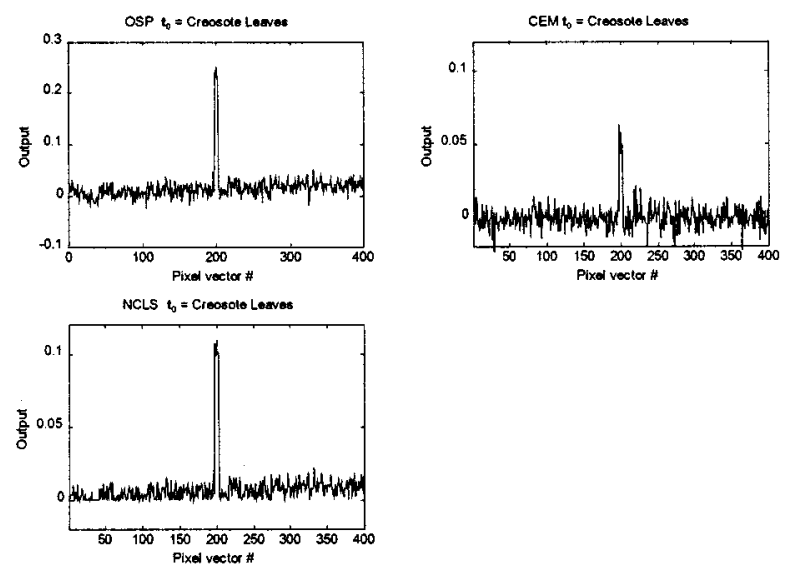

(a)
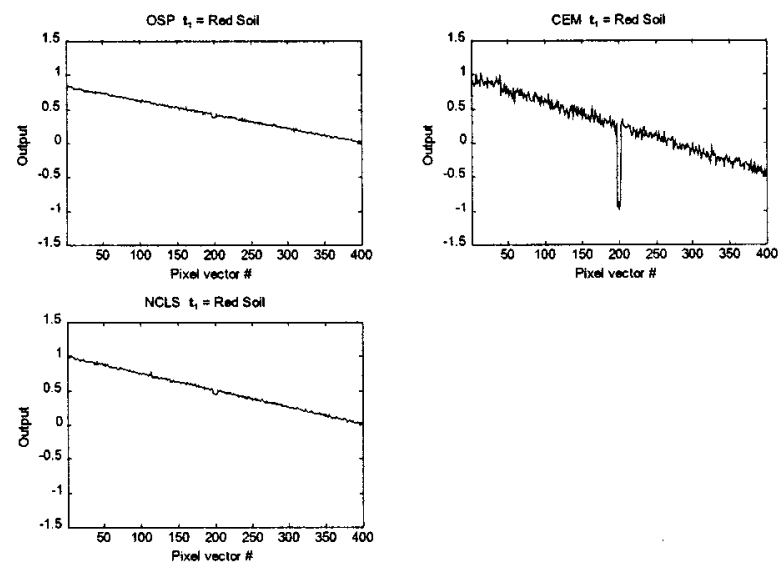

(b)
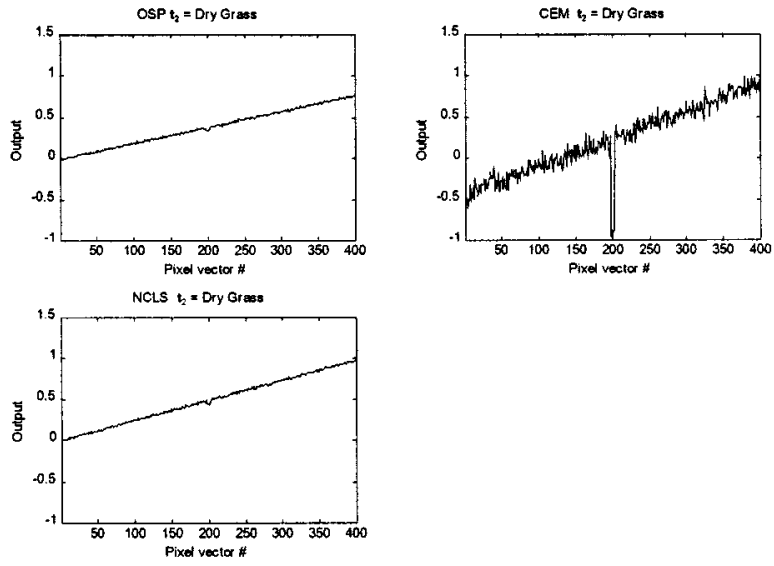

(c)

Fig. 6. (a)-(c) Comparative results of UNCLS, CEM, and OSP, with targets generated by UNCLS and the inital target $\boldsymbol{T}_{0}$, given by the desired creosote leaves signature.

sults of NCLS, CEM and OSP in detection of creosote leaves. Unlike Fig. 3, the performances of three methods are quite different. OSP produced the worst performance because the dimensionality of orthogonal subspace used for detection of creosote leaves had been reduced by two due to an addition of the black-
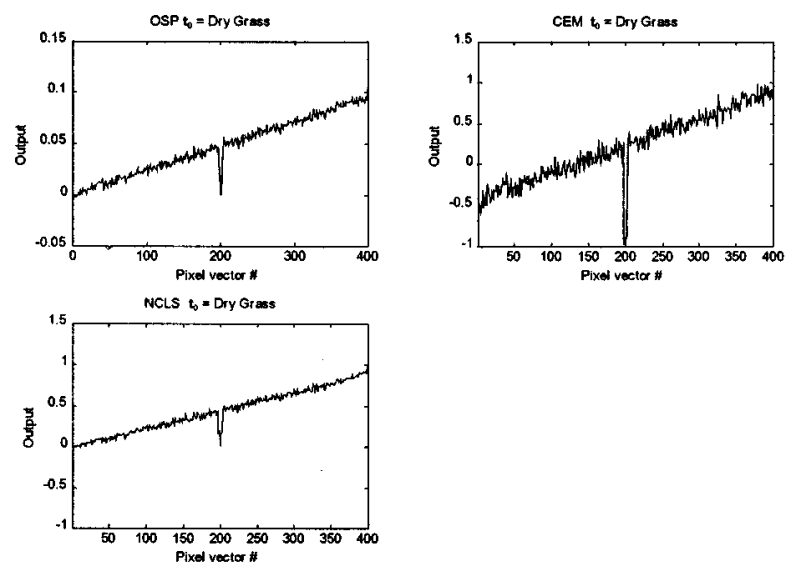

(a)
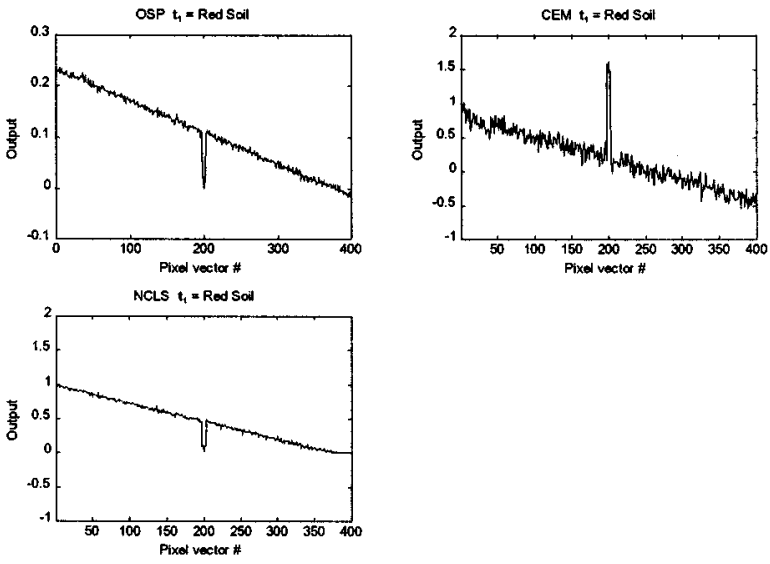

(b)
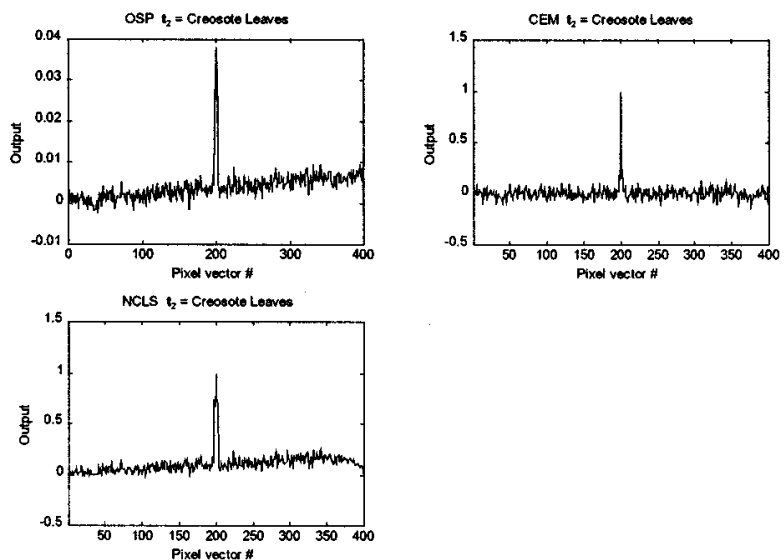

(c)

Fig. 7. (a)-(c) Comparative results of UNCLS, UCEM, and UOSP with no prior target knowledge.

brush and sagebrush signatures. As a result, its detection capability was considerably deteriorated by the undesired signature annihilator $P_{\boldsymbol{U}}^{\perp}$, since the spectra of the interferers blackbrush and sagebrush are very similar to that of creosote leaves. Similarly, NCLS also suffered from the same problem, which resulted in slight degradation in detection of creosote leaves at 


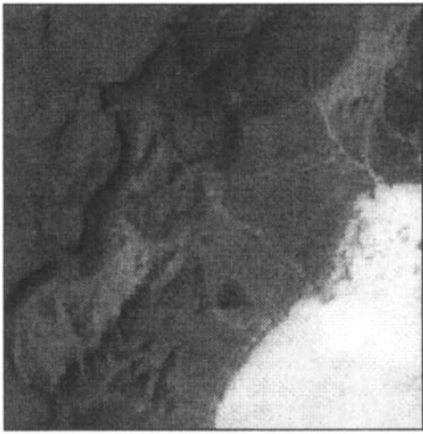

Fig. 8. AVIRIS image scene.

pixel 200, but it actually did better than it did for Fig. 3(c) at other pixels in terms of nulling the abundance of creosote leaves. For CEM, the result was identical to that in Fig. 3, because the addition of blackbrush and sagebrush with zero abundance did not affect the output energy of the CEM filter. This example also demonstrates a major difference between NCLS and CEM.

2) Sensitivity to Target Knowledge: In Section VI-A, the complete knowledge of target signatures described was assumed in Examples 1-3 to demonstrate how crucial the number of eigenvectors used in computation of $R_{L \times L}^{-1}$ are. In this section, the sensitivity issue of the precise knowledge of target signatures used in NCLS, CEM, and OSP will be investigated.

Example 4: Partial Knowledge of Target Signature: Once again, the same simulated data used in Example 2 were considered to demonstrate how partial target knowledge is utilized in UNCLS. Here, the only knowledge available to us was that there was a signature of creosote leaves present in the data. Following the UNCLS algorithm, the creosote leaves signature was set to the initial target signature $\boldsymbol{t}_{0}$. The NCLS algorithm was used to estimate the abundance fraction of $\boldsymbol{t}_{0}$, denoted by $\hat{\alpha}_{0}^{(1)}$, in each of 400 simulated pixel vectors, where the $\boldsymbol{r}$ is dropped from the notation of $\hat{\alpha}_{0}^{(1)}$ to simplify notations, i.e., $\hat{\alpha}_{0}^{(1)} \equiv \hat{\alpha}_{0}^{(1)}(\boldsymbol{r})$. Using $\hat{\alpha}_{0}$, we calculated the LSE between all simulated pixel vectors and $\hat{\alpha}_{0}^{(1)} \boldsymbol{t}_{0}$. Since the resulting maximum LSE was not below a prescribed threshold, the UNCLS was continued to find a pixel vector that yielded the maximum LSE. In this example, the seventh pixel vector with $98.5 \%$ red soil was selected as the first target, denoted by $\boldsymbol{t}_{1}$. The UNCLS algorithm was then used to estimate the abundance fractions of $\boldsymbol{t}_{0}$ and $\boldsymbol{t}_{1}$, denoted by $\hat{\alpha}_{0}^{(2)}$ and $\hat{\alpha}_{1}^{(2)}$. Using the estimated abundance fractions, we calculated LSE of all simulated pixels between the least squares linear mixture $\hat{\alpha}_{0}^{(2)} \boldsymbol{t}_{0}+\hat{\alpha}_{1}^{(2)} \boldsymbol{t}_{1}$. Because the resulting maximum LSE was still not below the prescribed threshold, the UNCLS algorithm was continued and the 400th pixel with $100 \%$ dry grass was selected as a second target $\boldsymbol{t}_{2}$. After finding $\boldsymbol{t}_{2}$, the resulting maximum LSE was below the prescribed threshold, and the UNCLS algorithm was terminated. At this point, we had generated two more target signatures, $\boldsymbol{t}_{1}=$ red soil and $\boldsymbol{t}_{2}=$ dry grass, which were not known a priori. Using these three target signatures $\boldsymbol{t}_{0}, \boldsymbol{t}_{1}$, and $\boldsymbol{t}_{2}$ as the signature matrix $\boldsymbol{M}$ for NCLS algorithm and OSP, the results are shown in Fig. 6(a)-(c), where creosote leaves, red soil, and dry grass were used as the desired signatures and detected, respectively. As we can see from Fig. 6, CEM performed very well by extracting all the three target signatures but did not detect correct amounts of target signatures. In addition, the fractions of abun-
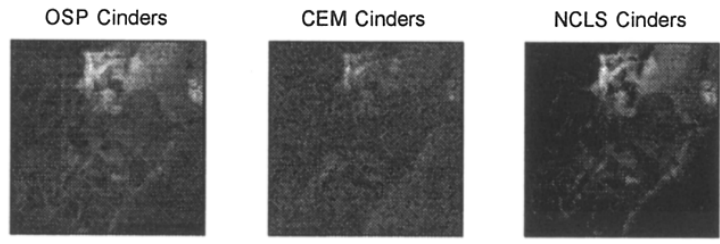

(a)
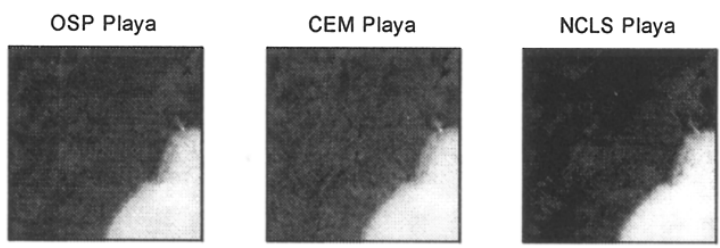

(b)
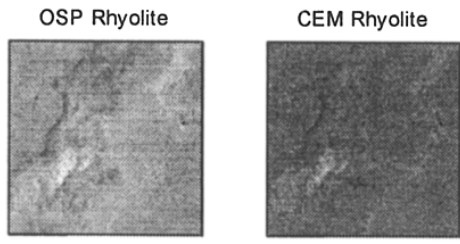

NCLS Rhyolite

(c)
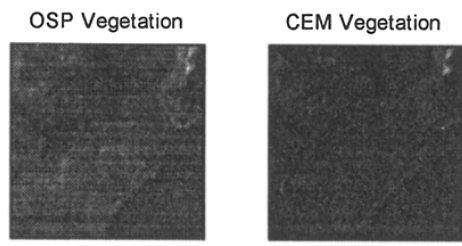

NCLS Vegetation

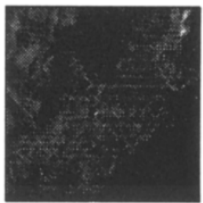

(d)
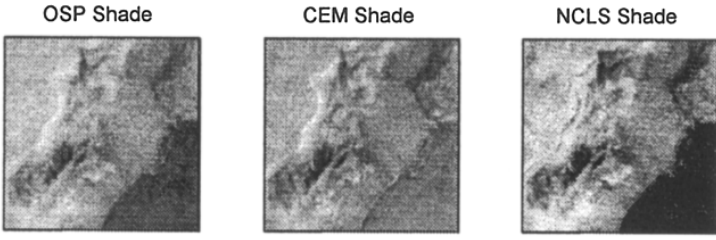

(e)

Fig. 9. Results of NCLS, OSP, and CEM, using $q=158$, where figures labeled by (a), (b), (c) (d), and (e) are detection results of cinders, playa, rhyolite, vegetation, and shade, respectively.

dance detected by CEM for these three desired signatures were very different. Except for creosote leaves, the CEM-detected abundance fractions for red soil and dry grass were negative, where their accurate amounts were supposed to be $45 \%$. This implies that CEM can detect anomalies even though it failed to detect the desired target signatures such as red soil and dry grass. Compared to CEM, UOSP and UNCLS detected more accurate abundance fractions for these three desired signatures. In particular, UNCLS produced almost correct amounts of abundance for all the three signatures. This example demonstrates two interesting facts. From a target detection's point of view, CEM performed better than OSP and UNCLS in terms of weak or 

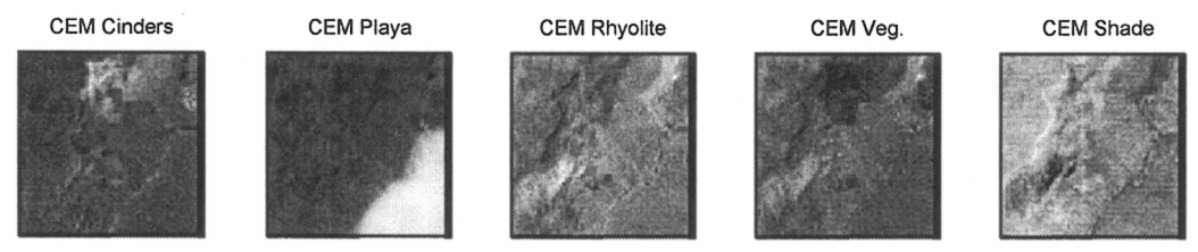

(a)
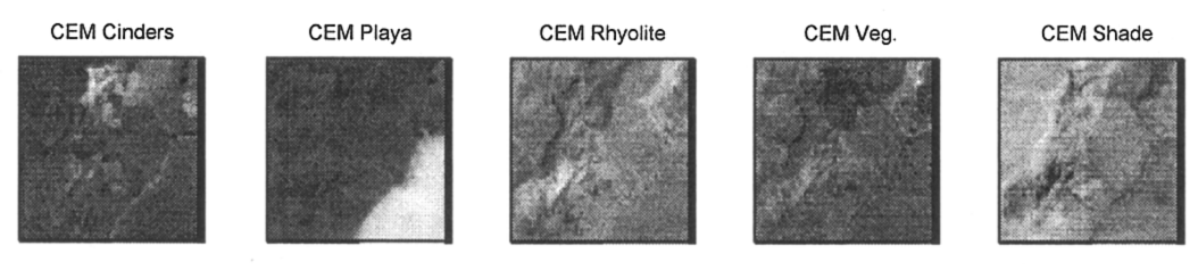

(b)
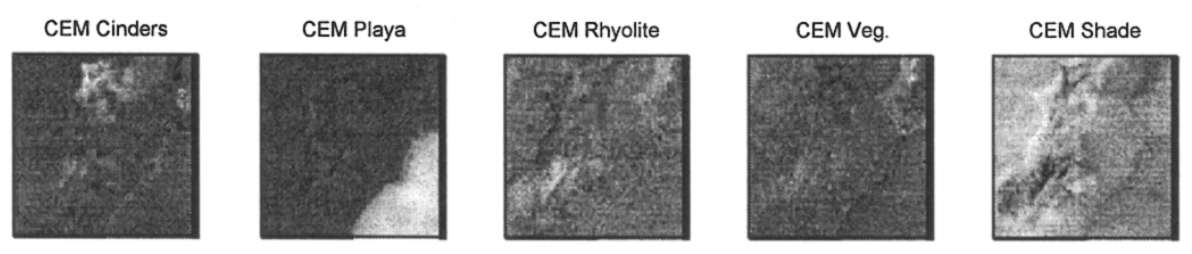

(c)
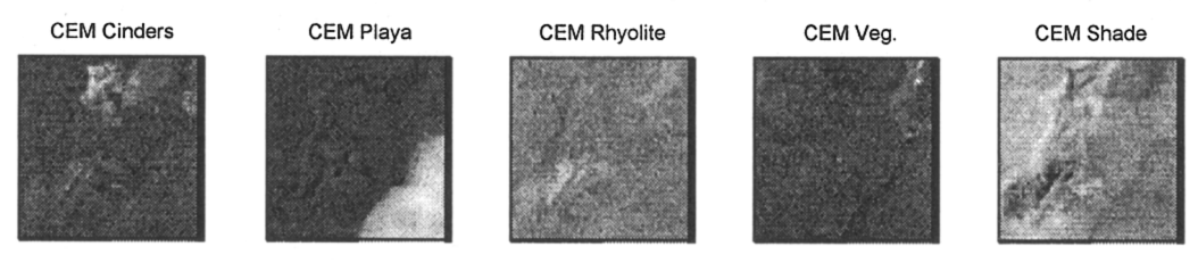

(d)
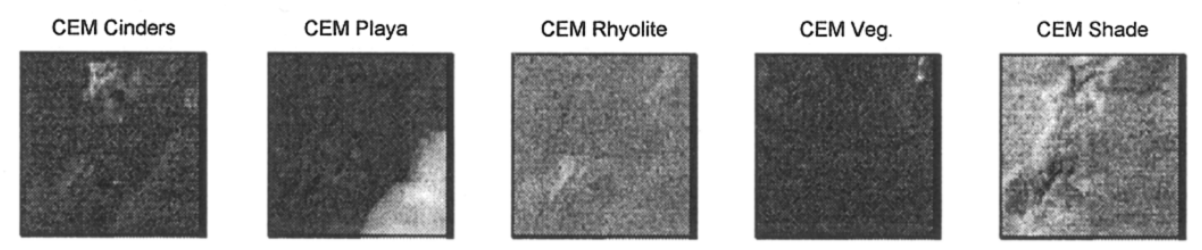

(e)

Fig. 10. Comparative results of CEM for cinders, playa, rhyolite, vegetation, and shade using: (a) $q=5$, (b) $q=10$, (c) $q=20$, and (d) $q=40$, and $q=80$.

small targets even though it may fail to detect right targets. On the other hand, from an abundance detection's point of view, UNCLS performed substantially better than UOSP and CEM. It is not only capable of detecting right target signatures but also estimating correct amounts of target abundance. UOSP seems right in between in either case.

Example 5: No Target Knowledge Available A Priori: The only difference between this example and Example 4 was that no initial target signature $\boldsymbol{t}_{0}$ was given a priori. Target $\boldsymbol{t}_{0}$ must be generated from the data set. In this case, we selected the pixel vector with maximum length, which turned out to be the 400th pixel vector with $100 \%$ dry grass. Using this pixel vector to initialize the UNCLS algorithm, and following the same procedure in Example 4, we found the fourth pixel vector to be $\boldsymbol{t}_{1}=$ redsoil with $99.25 \%$, and the 200th pixel vector $\boldsymbol{t}_{2}=$ creosote leaves with $10 \%$ and the UNCLS was terminated. The 

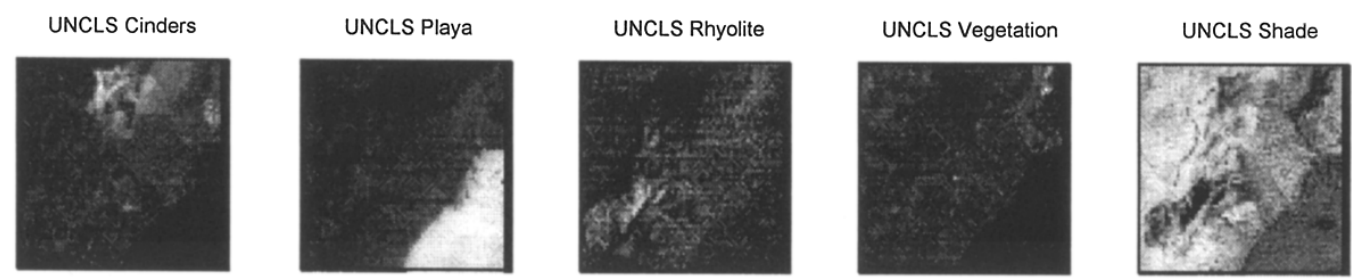

Fig. 11. Results of UNCLS for cinders, playa, rhyolite, vegetation, and shade with partial target knowledge, where for each image only the target of interest was known a priori.
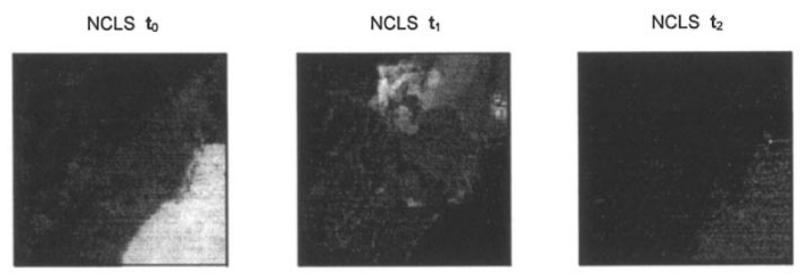

NCLS $t_{3}$

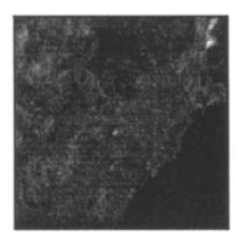

NCLS t4

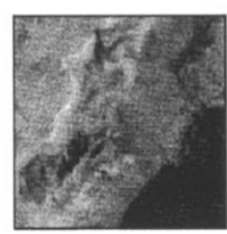

NCLS $t_{5}$

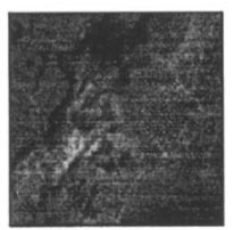

Fig. 12. Results of UNCLS with no target knowledge.

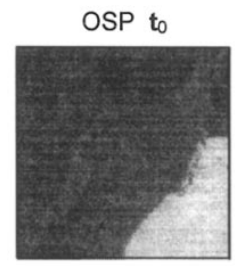

OSP $t_{3}$

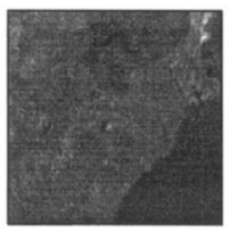

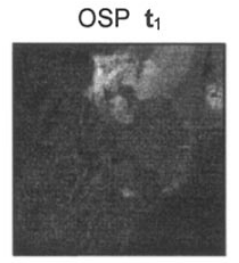

OSP $t_{4}$

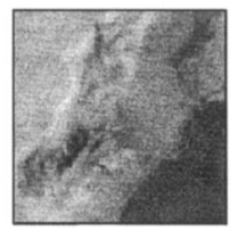

OSP $t_{2}$

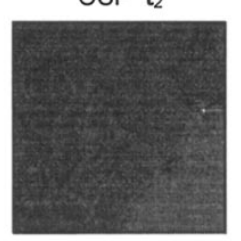

OSP $t_{5}$

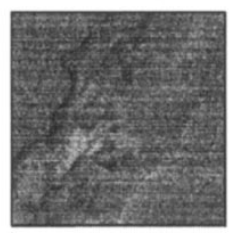

Fig. 13. Results of UOSP using targets generated by UNCLS.

procedure used to generate these three signatures was also used to extend CEM and OSP to UCEM and UOSP. It should be noted that targets generated by UNCLS in this example were different from those generated in Example 4. Fig. 7(a)-(c) show the detection results produced by UNCLS, UCEM, and UOSP, respectively. Interestingly, the detection result of red soil in Fig. 7(b) produced by UCEM looked exactly upside down compared to that in Fig. 6(b), produced by UCEM with slightly different magnitudes. For detection of creosote leaves, the results produced by UCEM in Figs. 6(a) and 7(c) looked similar, but the detected abundance fractions were different. For detection of dry grass, UCEM produced nearly the same results in both cases. Like Example 4, UCEM can be only used for target detection purpose. Comparing the results in Fig. 7 generated by UOSP and UNCLS against those in Fig. 6, UNCLS, and UOSP did
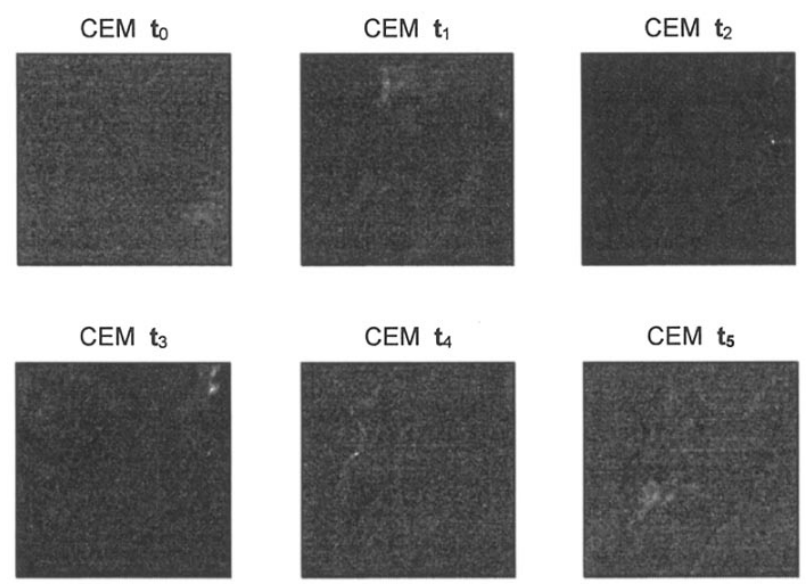

Fig. 14. Results of UCEM using targets generated by UNCLS with $q=158$.

not perform as well as they did in Fig. 6 in terms of estimating abundance fractions due to the lack of prior information about target signatures. Under this circumstance, UOSP and UNCLS behaved more like a target detector rather than a target abundance estimator, as shown in Fig. 6.

\section{B. Hyperspectral Images}

The hyperspectral data used in the following experiments are AVIRIS data, which were the same data in [15]. It is a subscene of $200 \times 200$ pixels extracted from the upper left corner of the Lunar Crater Volcanic Field, Northern Nye County, NV, shown in Fig. 8, where five target signatures of interest are cinders, rhyolite, playa (dry lakebed), vegetation, and shade. Fig. 9 shows the results of NCLS, OSP, and CEM using the number of eigenvectors $q=158$, where figures labeled by (a), (b), (c), and (d) show cinders, playa, rhyolite, and vegetation as targets respectively, and figures labeled by (e) are results of the shade. From these images, we see that NCLS performed the best in all cases. In order to see how the number of eigenvectors $q$ used in CEM affects its performance, five more different numbers $q=5,10,20,40,80$ were also used in CEM implementation, and the results are shown in Fig. 10. As we can see, CEM performed well in detection of cinders, rhyolite, and shade when the value of $q$ was small. On the contrary, CEM did a better job in the detection of vegetation if a large value of $q$ was used. This phenomenon coincides with the conclusion made in Example 2 of Section VI-A.

In the above experiment, we assumed that the complete knowledge of all five target signatures was known a priori. In the following experiment, we assume that partial target knowledge is available. In this case, only one of five signatures 

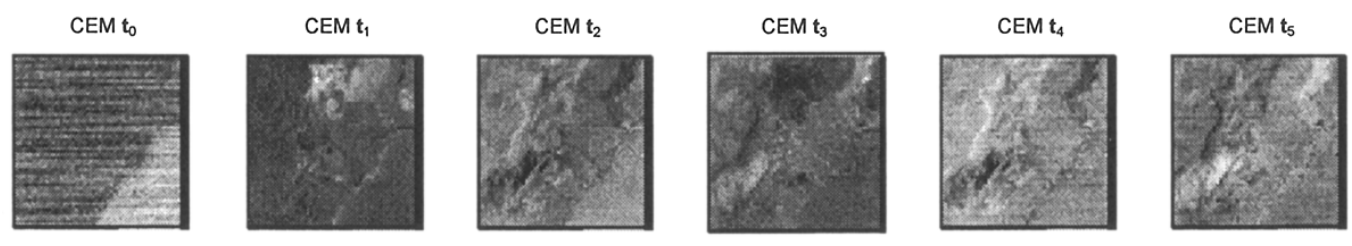

(a)
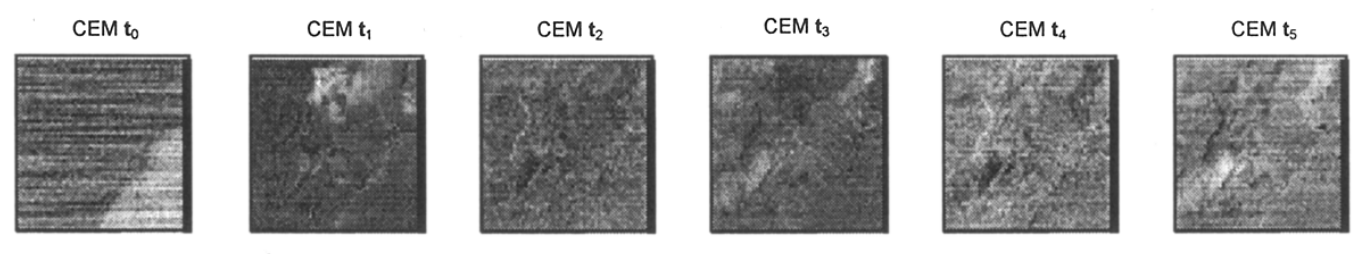

(b)
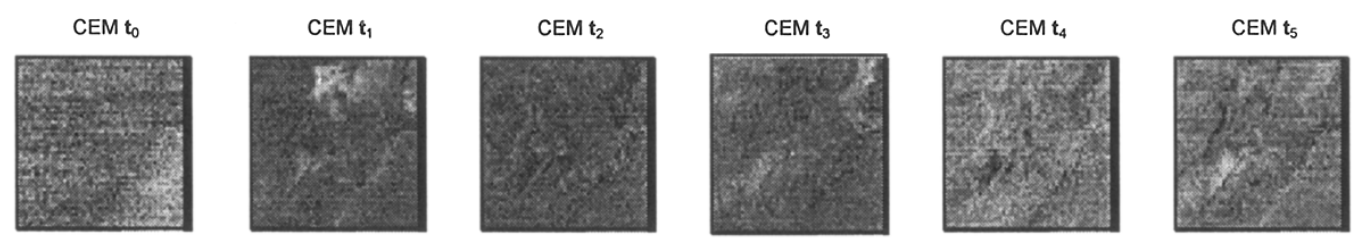

(c)
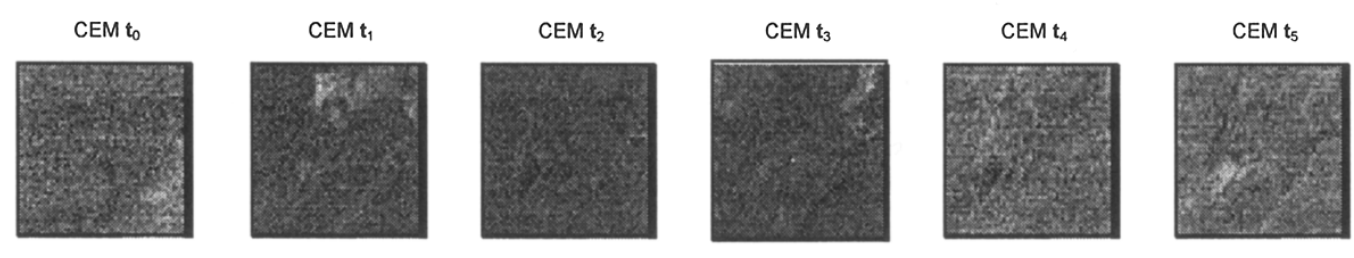

(d)
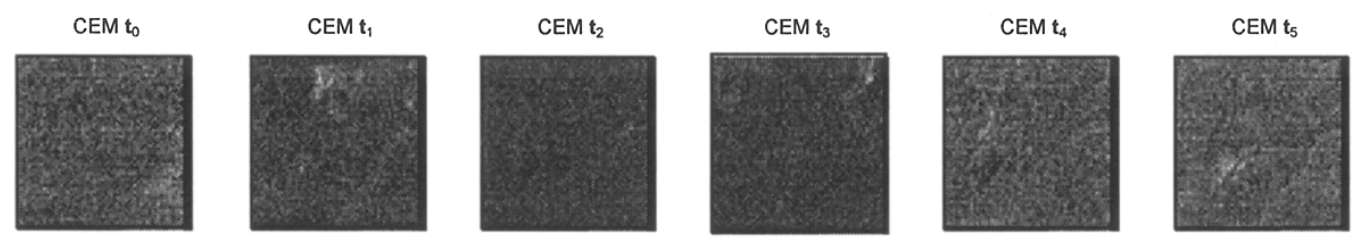

(e)

Fig. 15. Comparative results of UCEM for cinders, playa, rhyolite, vegetation, and shade, using (a) $q=5$, (b) $q=10$, (c) $q=20$, and (d) $q=40$, and $q=80$.

is known a priori. UNCLS is used for target detection. The results are shown in Fig. 11 and are comparable to those in Fig. 9. Finally, we conclude the experiment by assuming that no prior target knowledge is given. In this situation, UNCLS generated six targets from the scene in Fig. 8, and they were detected and classified in Fig. 12. The results are also very similar to those in Figs. 9 and 11 , where $\boldsymbol{t}_{0}=$ playa, $\boldsymbol{t}_{1}=$ cinders, $t_{2}=$ anomalous target, $t_{3}=$ vegetation, $t_{4}=$ shade, and $\boldsymbol{t}_{5}=$ rhyolite. Interestingly, the third generated target $\boldsymbol{t}_{3}$ was an edge pixel of the dry lakebed. This pixel vector can be thought of as an anomalous target and was not picked up in previous experiments. This experiment further demonstrates the potential usefulness of UNCLS, which can be used for detection of anomalies, a task that supervised NCLS and 
CEM cannot achieve. Figs. 13 and 14 were also generated by UOSP and UCEM with $q=158$ using the targets generated by UNCLS. As we can see from Fig. 14, without precise target signature knowledge, UCEM performed very poorly except at detecting vegetation. Fig. 15 shows the results of UCEM using $q=5,10,20,40,80$, as was done in Fig. 10, produced by CEM. Obviously, UCEM performed much worse than its counterparts produced by CEM in Fig. 10. This example shows that the success of CEM was completely determined by the knowledge of the desired target signature and the number of eigenvectors $q$ used in CEM.

\section{CONCLUSION}

In this paper, a target abundance-constrained subpixel target detection approach, NCLS, is introduced. It is a least squares method based on a nonnegativity abundance-constrained linear spectral mixture model. Since there is no closed form that can be derived for optimal solutions, a fast, efficient numerical algorithm is developed to generate a desired optimal subpixel detector. The NCLS-based detector is then further extended to an unsupervised version where no prior information is required. Despite the success of CEM in hyperspectral image classification, the strengths and weaknesses of CEM have not been investigated in depth previously in the literature. This paper presents a comprehensive study of CEM and also conducts a comparative analysis among the target abundance-constrained NCLS, the target signature-constrained CEM, and the unconstrained OSP. As shown in the experiments, NCLS generally performs subpixel detection significantly better than the unconstrained OSP. Both NCLS and CEM have been shown to have their advantages and disadvantages (see Appendix). If there are small targets in an image scene, CEM generally performs better than NCLS and OSP. This may explain why CEM has achieved much success in HYDICE data analysis due to their significantly improved spatial resolution. On the other hand, NCLS performs much better when no precise target knowledge is available or when targets are relatively large or ubiquitous in the scene, such as background signatures. Since CEM uses the sample correlation matrix to calculate the optimal weight vector in (17), it is actually a spatial filter and can be viewed as a spatial analysis technique. This is the reason the rank of the sample correlation matrix plays a significant role in performance. In contrast to CEM, NCLS does not deal with spatial correlation but only spectral correlation among target signatures. Therefore, NCLS is a spectral analysis technique. From this point of view, both techniques are completely different. Nevertheless, NCLS seems to be more robust and less sensitive to noise and target knowledge than CEM.

As a concluding remark, the NCLS algorithm has been shown to be converge in all the experiments conducted in this paper. However, it should be noted that on some occasions, the NCLS algorithm could oscillate between two passive sets since it steers back to a zero passive set, which usually contains more than one negative component during each iteration. Should it occur, the NCLS algorithm would adjust only one component at a time during each iteration. More details about the analysis of the NCLS algorithm can be found in [28].

\section{APPENDIX}

In this appendix, we list possible advantages and disadvantages of three methods studied in this paper: OSP, CEM, and NCLS. It is by no means a comprehensive list. All advantages and disadvantages described below are based on experiments conducted in our lab and reflect only our lab's opinions.

\section{OSP \\ - Advantages \\ 1) It is simple, efficient, and effective when target sig- natures are distinct. \\ 2) It is only based on second-order statistics and does not make Gaussian noise assumption. \\ - Disadvantages \\ 1) It is unconstrained and does not yield optimal solu- tions. \\ 2) It requires the knowledge of target signatures and a linear spectral mixture model. \\ 3) It can only be used for target detection and cannot detect similar targets effectively. \\ 4) It cannot be used for material quantification.}

\section{CEM}

- Advantages

1) It improves unconstrained linear unmixing methods and can be implemented in real time processing.

2) No a priori knowledge except the desired target signature is required.

3) No linear mixture model is assumed.

4) It is very efficient and can detect small targets very effectively.

- Disadvantages

1) It is very sensitive to noise and the desired target signature knowledge.

2) It can only detect one target at a time and cannot detect similar targets effectively.

3) It requires the rank of the sample correlation matrix to achieve the best results and separate images to classify multiple targets.

4) It cannot be used for material quantification.

\section{$N C L S$}

- Advantages

1) It improves unconstrained linear unmixing methods and can be implemented in an unsupervised manner.

2) It can detect similar targets as well as multiple targets.

3) It is not as sensitive as CEM to target signature knowledge.

4) It can be extended to an unsupervised version, UNCLS.

\section{- Disadvantages}

1) It requires a linear spectral mixture model.

2) It is not as effective as CEM if targets are small and the target knowledge is accurate.

3) It cannot be used for material quantification. 


\section{ACKNOWLEDGMENT}

The authors wish to thank Dr. Harsanyi for providing hyperspectral data set used for experiments in this paper.

\section{REFERENCES}

[1] D. E. Sabol, J. B. Adams, and M. O. Smith, "Quantitative sub-pixel spectral detection of targets in multispectral images," J. Geophys. Res., vol. 97, pp. 2659-2672, 1992

[2] M. O. Smith, J. B. Adams, and D. E. Sabol, "Spectral mixture analysis-new strategies for the analysis of multispectral data," in Image Spectroscopy-A Tool for Environmental Observations, J. Hill and J. Mergier, Eds. Brussels, Belgium: ECSC, EEC, EAEC, 1994, pp. 125-143.

[3] J. B. Adams, M. O. Smith, and A. R. Gillespie, "Image spectroscopy: Interpretation based on spectral mixture analysis," in Remote Geochemical Analysis: Elemental and Mineralogical Composition, C. M. Pieters and P. A. Englert, Eds. Cambridge, U.K.: Cambridge Univ. Press, 1993 pp. $145-166$.

[4] A. R. Gillespie, M. O. Smith, J. B. Adams, S. C. Willis, A. F. Fischer III, and D. E. Sabol, "Interpretation of residual images: Spectral mixture analysis of AVIRIS images," in Proc. 2nd AVIRIS Workshop, Owens Valley, CA, 1990, pp. 243-270.

[5] J. B. Adams, M. O. Smith, and A. R. Gillepie, "Simple models for complex natural surfaces: A strategy for hyperspectral era of remote sensing," in Proc. IEEE Int. Geoscience and Remote Sensing Symp. '89, 1989, pp. 16-21.

[6] S. Tompkins, J. F. Mustarrd, C. M. Pieters, and D. W. Forsyth, "Optimization of endmembers for spectral mixture analysis," Remote Sens. Environ., vol. 59, pp. 472-489, 1997.

[7] J. W. Boardman, "Inversion of imaging spectrometry data using singular value decomposition," in Proc. IEEE Symp. Geoscience and Remote Sensing, 1989, pp. 2069-2072.

[8] J. J. Settle and N. A. Drake, "Linear mixing and estimation of ground cover proportions," Int. J. Remote Sensing, vol. 14, no. 6, pp. 1159-1177, 1993.

[9] Y. E. Shimabukuro and J. A. Smith, "The least-squares mixing models to generate fraction images derived from remote sensing multispectral data," IEEE Trans. Geosci. Remote Sensing, vol. 29, pp. 16-20, Jan. 1991.

[10] M. O. Smith, D. A. Roberts, J. Hill, W. Mehl, B. Hosgood, J. Verdebout, G. Schmuck, C. Koechler, and J. B. Adams, "A new approach to quantifying abundances of materials in multispectral images," in Proc. IEEE Int. Geoscience and Remote Sensing Symp. '94, Pasadena, CA, August 1994, pp. 2372-2374.

[11] J. W. Boardman, "Leveraging the high dimensionality of AVIRIS data for improved sub-pixel target unmixing and rejection of false positive: Mixture tuned matched filtering," Proc. 7th Annu. JPL Earth Science Workshop, 1998.

[12] J. C. Harsanyi, "Detection and classification of subpixel spectral signatures in hyperspectral image sequences," Ph.D. dissertation, Dept. Elect. Eng., Univ. Maryland, Baltimore County, 1993.

[13] E. A. Ashton and A. Schaum, "Algorithms for the detection of sub-pixel targets in multispectral imagery," Photogramm. Eng. Remote Sensing, pp. 723-731, July 1998 .

[14] D. Heinz, C.-I Chang, and M. L. G. Althouse, "Fully constrained least squares-based linear unmixing," in Int. Geoscience and Remote Sensing Symp. '99, Hamburg, Germany, June 28 - July 21999.

[15] J. C. Harsanyi and C.-I Chang, "Hyperspectral image classification and dimensionality reduction: An orthogonal subspace projection," IEEE Trans. Geosci. Remote Sensing, vol. 32, pp. 779-785, July 1994.

[16] C.-I Chang, T.-L. E. Sun, and M. L. G. Althouse, "An unsupervised interference rejection approach to target detection and classification for hyperspectral imagery," Opt. Eng., vol. 37, no. 3, pp. 735-743, March 1998.

[17] C. L. Lawson and R. J. Hanson, "Solving least squares problems," in Proc. Classics in Applied Mathematics. Philadelphia, PA, 1995.

[18] R. Bro and S. D. Jong, "A fast nonnegativity-constrained least squares algorithm," J. Chemom., vol. 11, pp. 393-401, 1997.

[19] J. C. Harsanyi, W. Farrand, and C.-I Chang, "Detection of subpixel spectral signatures in hyperspectral image sequences," in Proc. American Society of Photogrammetry and Remote Sensing, Reno, NV, 1994, pp. 236-247.
[20] W. W. Farrand and J. C. Harsanyi, "Mapping the distribution of mine tailing in the coeur d'Alene River Valley, Idaho, through the use of constrained energy minimization technique," Remote Sens. Environ., vol. 59, pp. 64-76, 1997.

[21] B. D. Van Veen and K. M. Buckley, "Beamforming: A versatile approach to spatial filtering," IEEE ASSP Mag., Apr. 4-24, 1988.

[22] S. Haykin, Adaptive Filter Theory, 3rd ed. Englewood Cliffs, NJ: Prentice-Hall, 1996.

[23] M. L. G. Althouse and C.-I Chang, "Chemical vapor detection with a multispectral thermal imager," Opt. Eng., vol. 30, no. 11, pp. 1725-1733, July 1991.

[24] O. L. Frost, III, "An algorithm for linearly constrained adaptive array processing," Proc. IEEE, vol. 60, pp. 926-935, 1972.

[25] C.-I Chang and H. Ren, "Linearly constrained minimum variance beamforming for target detection and classification in hyperspectral imagery," in Int. Geoscience and Remote Sensing Symp. '99, Hamburg, Germany, June 28 - July 21999.

[26] J. Harsanyi, W. Farrand, and C.-I Chang, "Determining the number and identity of spectral endmembers: An integrated approach using Neyman-Pearson eigenthresholding and iterative constrained RMS error minimization," in Proc. 9th Thematic Conf. Geologic Remote Sensing, Pasadena, CA, 1993

[27] C.-I Chang and Q. Du, "A noise subspace projection approach to determination of intrinsic dimensionality of hyperspectral imagery," in EOS/SPIE Symp. Remote Sensing, Conf. Image and Signal Processing for Remote Sensing V, Florence, Italy, September 20-24, 1999.

[28] D. Heinz and C.-I Chang, Constrained Least Squares Methods for Material Detection and Quantification of Linearly Mixing Problems, Disclosure and Invention. Baltimore, MD: Univ. Maryland, Baltimore County, May 1999.

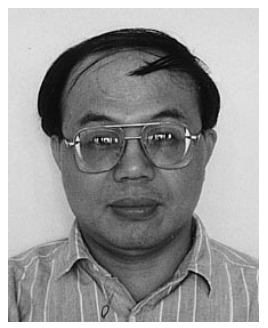

Chein-I Chang (S'81-M'87-SM'92) received the B.S., M.S., and M.A. degrees from Soochow University, Taipei, Taiwan, R.O.C., in 1973, the Institute of Mathematics at National Tsing Hua University, Hsinchu, Taiwan, in 1975, and the State University of New York, Stony Brook, in 1977, respectively, all in mathematics, and the M.S. and M.S.E.E. degrees from the University of Illinois, Urbana, in 1982. $\mathrm{He}$ then received the $\mathrm{Ph} . \mathrm{D}$. in electrical engineering from the University of Maryland, College Park, in 1987.

He was a Visiting Assistant Professor from January 1987 to August 1987, Assistant Professor from 1987 to 1993, and is currently an Associate Professor, Department of Computer Science and Electrical Engineering, University of Maryland, Baltimore County, Baltimore. He was a Visiting Specialist in the Institute of Information Engineering at the National Cheng Kung University, Tainan, Taiwan, from 1994 to 1995 . He is currently on the editorial board of Journal of High Speed Networks and the Guest Editor of a special issue on Telemedicine and Applications. His research interests include automatic target recognition, multispectral/hyperspectral image processing, medical imaging, information theory and coding, signal detection and estimation, and neural networks.

Dr. Chang is a member of SPIE, INNS, Phi Kappa Phi, and Eta Kappa Nu.

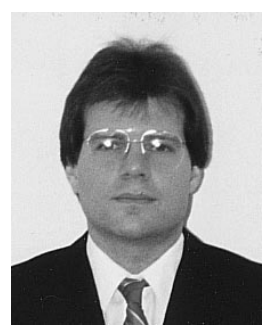

Daniel C. Heinz (S'92) received the B.S. and M.E.S. degrees in electrical engineering from the State University of New York, Buffalo, and Loyola College, Baltimore, MD, in 1991 and 1994, respectively. He is currently a Ph.D. degree candidate in the Department of Computer Science and Electrical Engineering, University of Maryland, Baltimore County (UMBC), Baltimore.

He worked on a chemical detector project with the United States Army Chemical and Biological Defense Command, Aberdeen Proving Ground, Aberdeen, MD, from 1991 to 1996. From 1996 to 1997, he worked on video teleconferencing at Tracor Applied Sciences, Inc., California, MD. From 1998 to 1999, he worked as a Teaching and Research Assistant with UMBC. Currently, he is an Instructor with UMBC. His research interests include digital signal processing, image processing, and remote sensing.

Mr. Heinz is a member of SPIE and Phi Kappa Phi. 